\title{
Intra- and Interchain Relaxation Processes in Meshlike Polymer Networks
}

\author{
Andrew A. Gurtovenko and Yuli Ya. Gotlib* \\ Institute of Macromolecular Compounds, Russian Academy of Sciences, Bolshoi Prospect 31, V. O., \\ St. Petersburg, 199004, Russia \\ Received J anuary 8, 1998; Revised Manuscript Recei ved May 8, 1998
}

\begin{abstract}
The dynamic properties of meshlike polymer networks are considered using the cubic network of multisegmental "bead and spring" Rouse chains. The normal mode treatment is used. Rigorous expressions for the mean-square displacements of elements of the Gaussian network (junctions and nonjunction beads) are obtained. The autocorrelation functions of chain vectors between neighboring nonjunction beads (subchain vectors) and of end-to-end chain vectors between neighboring junctions are cal culated. The time dependence of the relaxation modulus of a network is also considered. Contributions of intra- and interchain relaxation processes to various dynamic characteristics of a Gaussian network are compared. The time behavior of dynamic quantities obtained is estimated for different scale motions. It is shown that the relaxation of regular polymer networks consisting of long multisegmental Gaussian chains can be considered as a superposition of four different types of relaxation processes. These are the relaxation of network chains with fixed junctions, the relaxation of chains with free ends, the relaxation processes in the intermediate region of the spectrum, and interchain cooperative network motions. The possibility of describing low-frequency dynamic properties of networks by a simplified coarse-grained network model used previously is proved.
\end{abstract}

\section{Introduction}

Network models with different topologies (treelike and meshlike) have been considered by many authors ${ }^{1-17}$ for describing dynamic relaxation properties of crosslinked polymers. In most previous theories, the chain fragment between the neighboring network junctions (cross-links) is modeled by a "bead and spring" Rouse chain. ${ }^{18,19}$ The fundamental feature of cross-linked polymers is the existence of cooperative chain motions on scales larger than the distance between neighboring network junctions. The cross-linking of polymer chains in the network leads to the splitting and broadening of the relaxation spectrum lines of a unique polymer chain and to the appearance of an additional branch representing the low-frequency collective spectrum. ${ }^{1-3,12}$

In the theoretical consideration of polymer network dynamics, the intra- and interchain spectra have usually been studied separately (except in refs 1, 3, and 12). It has been suggested that the intrachain motions on scales smaller than the distance between neighboring junctions (cross-links) can be satisfactorily described by the relaxation behavior of a chain that is not included in the network structure. For example, for describing the viscoelastic properties of cross-linked polymers in the high-frequency domain, the multisegmental Gaussian chain with fixed ends in space has been used. 19,20 To describe the low-frequency interchain branch of the relaxation spectrum, the simplified coarse-grained dynamic model of polymer networks has usually been considered. ${ }^{4-11,13-17}$ In this dynamic model, the multisegmental Gaussian chain between neighboring junctions of a network is modeled by a single spring with the elasticity constant corresponding to that of a multisegmental chain. All dissi pation effects related to the friction of network chains with respect to the external viscous medium are described by the friction of network

* Author to whom correspondence should be addressed. Telephone: 7-(812)-218-5601. Fax: 7-(812)-218-6869. E-mail: yygotlib@macro.lgu.spb.su. junctions. The friction constant for a junction of the coarse-grained network model is determined by the friction of chain fragments directly attached to a given network junction. Therefore, the simplified coarsegrained model of a polymer network describes only the collective motions of network chains on scales larger than the average distance between cross-links.

In the above mentioned "separate" description of relaxation properties, the dynamic behavior of the polymer network in the intermediate region between intra- and interchain motions has not been treated in detail. However, just this intermediate region, the intrachain relaxation spectrum, and a small initial part of interchain network spectrum are usually available in real experiments and computer simulation if the time range studied is not much greater than the maximum relaxation time of the chain between the junctions. Therefore, the relaxation properties in the intermediate domain of the spectrum are of great interest in crosslinked polymer dynamics. Moreover, the relaxation dynamic properties of a chain included in the network structure and those of a linear polymer chain have to differ with increasing scales of intrachain motions.

To take into account the intrachain motions in a more rigorous way, it is necessary to consider the dynamic model of a network consisting of polymer chains containing a large number of Gaussian subchains. The case of a meshlike network of long, flexible Gaussian chains has been considered by Ham. ${ }^{1}$ The distribution of relaxation times of the polymer network was obtained, which permits the calculation of the energy stored in steady state shear. It has been shown ${ }^{1}$ that the distribution of relaxation times changes at times greater than the longest relaxation time of a chain between cross-links. However, the local dynamic properties of network chains on scales with an order of magnitude equal to the dimension of the network cell have not been considered in ref 1 . The network of multisegmental "bead and spring" Rouse chains has been applied by Gotlib and Salikhov ${ }^{3}$ for meshlike networks and by 


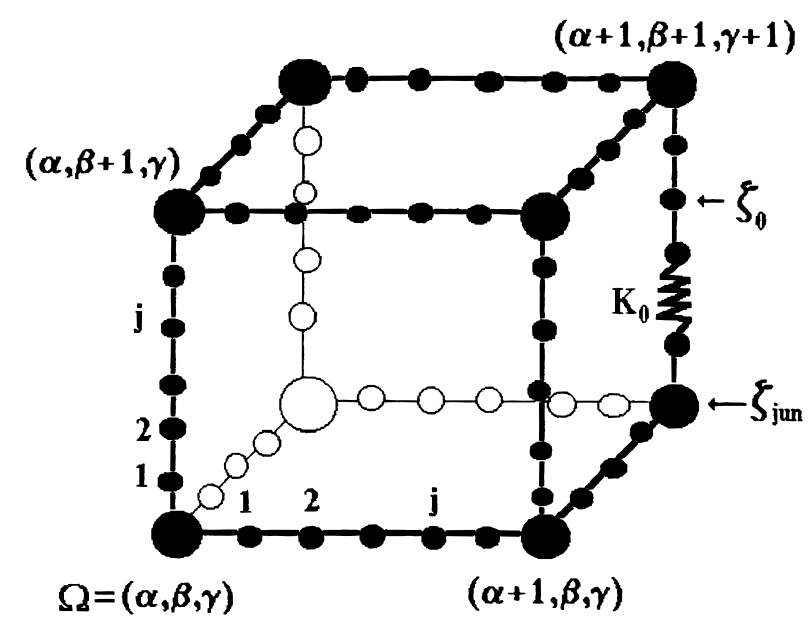

Figure 1. Elementary cell of a cubic network consisting of multisegmental "bead and spring" chains.

Kloczkowski, Mark, and Frisch ${ }^{12}$ for treelike networks. In these papers the relaxation times of cross-linked systems were calculated and the existence of two types of relaxation spectra (intra- and interchain spectra) was shown. The set of relaxation times obtained was used for calculating the dynamic modulus ${ }^{12}$ and viscosity. ${ }^{3}$ However, the dynamic characteristics of junctions and nonjunction elements of a meshlike network have not been studied previously. 1,3,12

This paper considers the dynamic properties of meshlike Gaussian networks in greater detail. Different local dynamic characteristics of network chains and junctions (cross-links), which have not been considered previously, will be obtained using a dynamic model of a regular cubic network of multisegmental Gaussian chains. ${ }^{3}$ All the normal modes of a cubic network consisting of multisegmental "bead and spring" Rouse chains will be obtained. This approach makes it possible to consider the dynamic characteristics of a network in intrachain, interchain, and intermediate regions of the relaxation spectrum. The paper is organized as follows. The mean-square displacements of junctions and nonjunction elements of network chains are calculated. The autocorrelation functions of the subchain vectors and of the end-to-end chain vectors between the neighboring network junctions (cross-links) are obtained. These autocorrelation functions can be manifested in the consideration of dielectric susceptibility of polymer networks containing polar macromolecules. ${ }^{15}$ The relaxation modulus of a Gaussian network is also calculated. The time behavior of dynamic characteristics obtained is estimated in various regions of the relaxation spectrum. A comparison of the contributions of intra- and interchain relaxation processes to the dynamic characteristics of the network is made. The lowfrequency dynamic properties of network junctions obtained are compared with the previous theories that have considered the simplified coarse-grained network model. A comparison of relaxation properties of a polymer chain embedded in the network structure with those of a linear polymer chain is discussed.

\section{Theory}

2.1. Dynamic Model of a Network. The cubic network of multisegmental Gaussian chains is considered (Figure 1). This network model is similar to that treated previously. ${ }^{3}$ An el ementary cell (or, a repeating unit) of a three-dimensional cubic network contains a junction and three "bead and spring" Rouse chains. 18 We consider a phantom Gaussian network; i.e., the excluded vol ume interactions and hydrodynamic interactions are neglected. The entanglement effects and the contributions of dangling chains are not discussed either. A regular network system is considered. Each Gaussian chain contains $n$ beads and, correspondingly, $(n+1)$ subchains connecting them. All subchains act as springs with the elasticity constant $\mathrm{K}_{0}$. It is assumed that the multisegmental chains of a given Gaussian network move inside the external viscous medium. This medium is a real solvent in the case of swollen networks. In the theory of dry polymer networks, the effective viscous medium mimics the friction effects due to the interactions between the network segments. ${ }^{13,16,17} \mathrm{~A}$ similar concept of effective medium has been taken into consideration in the study of dynamic properties of melts. ${ }^{21}$ The friction constants of a network junction and of a nonjunction bead are denoted as $\xi_{\text {jun }}$ and $\xi_{0}$, respectively. The dynamic behavior of a Gaussian network in the absence of external forces is studied. Only the $X$ projections of position vectors of the network elements will betreated because $X, Y$, and $Z$ projections for a Gaussian chain are independent of each other. Each cell of a cubic network $\Omega=(\alpha, \beta, \gamma)$ is marked by indices $\alpha, \beta$, and $\gamma$ (Figure 1 ). A network containing $\mathrm{N}$ junctions (cross-links) along each network direction (indices $\alpha, \beta$, and $\gamma$ change from 1 to $\mathrm{N}$ ) is considered. The total number of cells in the network system is equal to $N^{3}$. Let $X_{p}(\Omega ; j ; t)$ and $X_{0}(\Omega ; t)$ be the $X$ coordinates of the nonjunction bead and the network junction, respectively. Here index $p$ means the number of the multisegmental chains in the cell $(p=1,2,3$ for the chains of a cubic network) and index $j$ means the position of a bead along the multisegmental chain $(j=1, \ldots, n$ for nonjunction beads and $\mathrm{j}=0$ for network junctions).

The motion of the $X$ coordinate of a nonjunction bead is governed by

$$
\begin{aligned}
\xi_{0} \frac{\mathrm{d}}{\mathrm{dt}} \mathrm{X}_{\mathrm{p}}(\Omega ; \mathrm{j} ; \mathrm{t})+\mathrm{K}_{0}\left[2 \mathrm{X}_{\mathrm{p}}(\Omega ; \mathrm{j} ; \mathrm{t})-\mathrm{X}_{\mathrm{p}}(\Omega ; \mathrm{j}+1 ; \mathrm{t})-\right. \\
\left.\mathrm{X}_{\mathrm{p}}(\Omega ; \mathrm{j}-1 ; \mathrm{t})\right]=\mathrm{F}_{\mathrm{Br}}
\end{aligned}
$$

where $\mathrm{F}_{\mathrm{Br}}$ is the stochastic Brownian force. The junction of a cubic network is connected by subchains to six neighboring nonjunction beads. The equation of motion for a network junction has the form

$$
\begin{array}{r}
\xi_{\text {jun }} \frac{\mathrm{d}}{\mathrm{dt}} \mathrm{X}_{0}(\alpha, \beta, \gamma ; \mathrm{t})+\mathrm{K}_{0}\left[6 \mathrm{X}_{0}(\alpha, \beta, \gamma ; \mathrm{t})-\mathrm{X}_{1}(\alpha, \beta, \gamma ; 1 ; \mathrm{t})-\right. \\
\mathrm{X}_{1}(\alpha-1, \beta, \gamma ; \mathrm{n} ; \mathrm{t})-\mathrm{X}_{2}(\alpha, \beta, \gamma ; 1 ; \mathrm{t})-\mathrm{X}_{2}(\alpha, \beta-1, \gamma ; \mathrm{n} ; \mathrm{t})- \\
\left.\mathrm{X}_{3}(\alpha, \beta, \gamma ; 1 ; \mathrm{t})-\mathrm{X}_{3}(\alpha, \beta, \gamma-1 ; \mathrm{n} ; \mathrm{t})\right]=\mathrm{F}_{\mathrm{Br}}(2)
\end{array}
$$

In the consideration of local dynamic characteristics, it is convenient to use a network system with periodic boundary conditions. These conditions mean that

$$
\begin{aligned}
& \mathrm{X}_{1}(0, \beta, \gamma ; \mathrm{n} ; \mathrm{t})=\mathrm{X}_{1}(\mathrm{~N}, \beta, \gamma ; \mathrm{n} ; \mathrm{t}) \\
& \mathrm{X}_{2}(\alpha, 0, \gamma ; \mathrm{n} ; \mathrm{t})=\mathrm{X}_{2}(\alpha, \mathrm{N}, \gamma ; \mathrm{n} ; \mathrm{t}) \\
& \mathrm{X}_{3}(\alpha, \beta, 0 ; \mathrm{n} ; \mathrm{t})=\mathrm{X}_{3}(\alpha, \beta, \mathrm{N} ; \mathrm{n} ; \mathrm{t})
\end{aligned}
$$

Using the periodic boundary conditions, the exact solution for a given mechanical system can be obtained at an arbitrary number of network junctions in the system. 
2.2. Normal Mode Treatment. The general form of transformation from Cartesian coordinates $X$ to normal coordinates $\mathrm{Q}$ (normal modes) has the following structure for network elements (nonjunction beads $\mathrm{X}_{\mathrm{p}}(\Omega ; \mathrm{j} ; \mathrm{t})$ and network junctions $\left.\mathrm{X}_{0}(\Omega ; \mathrm{t})\right)$

$$
\begin{aligned}
& \mathrm{X}_{\mathrm{p}}(\Omega ; \mathrm{j} ; \mathrm{t})=\sum_{\theta, \psi} \operatorname{exp~i}[\vec{\Omega} \vec{\theta}]\left(\mathrm{A}_{\mathrm{p}} \sin \mathrm{j} \psi+\right. \\
& \left.\mathrm{B}_{\mathrm{p}} \cos \mathrm{j} \psi\right) \mathrm{Q}(\vec{\theta} ; \psi ; \mathrm{t}) \\
& \mathrm{X}_{0}(\Omega ; \mathrm{t})=\sum_{\vec{\theta}, \psi} \exp \mathrm{i}[\vec{\Omega} \vec{\theta}] \mathrm{B}_{0} \mathrm{Q}(\vec{\theta} ; \psi ; \mathrm{t})
\end{aligned}
$$

Here $\vec{\theta}=\left(\theta_{1}, \theta_{2}, \theta_{3}\right)$ is the network wave vector determining the phase shift between displacements of the neighboring network cells for a given normal mode. The quantity $\psi$ is the intrachain wave vector describing the phase shift between neighboring beads in the network chain. The time dependence of each normal mode has the form $\mathrm{Q}(\mathrm{t})=\mathrm{Q}(0) \exp [-\mathrm{t} / \tau]$ where $\tau$ is the relaxation time of a given normal mode. With the normal mode transformation (eq 4a), the equations of motion of nonjunction beads (eq 1) give the set of relaxation times $\tau$

$$
\tau(\psi)=\frac{\zeta_{0}}{\mathrm{~K}_{0} 2(1-\cos \psi)}
$$

Equation 5 for relaxation times of the polymer network has the same form as that for a linear Gaussian chain, ${ }^{18,19,21}$ but the intrachain wave vector of a network depends on the phase shift $\vec{\theta}$ between the network cells (see below eq 11).

Substituting transformation (eq 4a) into the periodic boundary conditions (eq 3 ) the components of the interchain wave vector can easily be obtained

$$
\theta_{1,2,3}=\frac{2 \pi}{N} S_{1,2,3} \quad S_{1,2,3}=0, \ldots, N-1
$$

In order to obtain the intrachain wave vector $\psi$ (and, thus, the relaxation times) and the transformation from Cartesian coordinates to normal modes, seven constants, $A_{1}, A_{2}, A_{3}, B_{0}, B_{1}, B_{2}$, and $B_{3}$, must be determined. These constants can be obtained by using the equation of motion of junction (eq 2) and the boundary conditions in the network junctions

$$
\begin{gathered}
\mathrm{X}_{0}(\alpha, \beta, \gamma ; \mathrm{t})=\mathrm{X}_{1}(\alpha-1, \beta, \gamma ; \mathrm{n}+1 ; \mathrm{t}) \\
\mathrm{X}_{0}(\alpha, \beta, \gamma ; \mathrm{t})=\mathrm{X}_{2}(\alpha, \beta-1, \gamma ; \mathrm{n}+1 ; \mathrm{t}) \\
\mathrm{X}_{0}(\alpha, \beta, \gamma ; \mathrm{t})=\mathrm{X}_{3}(\alpha, \beta, \gamma-1 ; \mathrm{n}+1 ; \mathrm{t}) \\
\mathrm{X}_{0}(\alpha, \beta, \gamma ; \mathrm{t})=\mathrm{X}_{1}(\alpha, \beta, \gamma ; 0 ; \mathrm{t}) \\
\mathrm{x}_{0}(\alpha, \beta, \gamma ; \mathrm{t})=\mathrm{X}_{2}(\alpha, \beta, \gamma ; 0 ; \mathrm{t}) \\
\mathrm{x}_{0}(\alpha, \beta, \gamma ; \mathrm{t})=\mathrm{X}_{3}(\alpha, \beta, \gamma ; 0 ; \mathrm{t})
\end{gathered}
$$

The exact analytical solution for a given mechanical system becomes possible only if the friction constant of a network junction is 3 times greater than that of a nonjunction bead ${ }^{3}$

$$
\xi_{\text {jun }}=3 \xi_{0}
$$

This corresponds to the superposition of beads (with friction constant $\xi_{0}$ ) of three intersecting multisegmental chains in the network junction. In this case the equation of motion of a network junction can be represented as the sum of three equations of motion for nonjunction beads. The combination of equations of motion (eq 2) with boundary conditions (eq 7) gives three different sets of wave vectors $\psi$ (cf. ref 3 )

$$
\begin{gathered}
\cos (\mathrm{n}+1) \psi_{1}=(1 / 3)\left(\cos \theta_{1}+\cos \theta_{2}+\cos \theta_{3}\right) \\
\sin (\mathrm{n}+1) \psi_{2}^{*}=0 \\
\sin (\mathrm{n}+1) \psi_{2}^{* *}=0
\end{gathered}
$$

Hence, for the sets of intrachain wave vectors $\psi$ we have

$$
\begin{gathered}
\psi_{1}=\frac{2 \pi}{\mathrm{n}+1} \mid \pm \frac{1}{\mathrm{n}+1} \arccos \frac{1}{3}\left(\cos \theta_{1}+\cos \theta_{2}+\right. \\
\left.\cos \theta_{3}\right) \quad I=0, \ldots, \mathrm{n} / 2 \\
\psi_{2}^{*}=\frac{\pi}{\mathrm{n}+1} \mathrm{k}_{2}^{*} \quad \mathrm{k}_{2}^{*}=1, \ldots, \mathrm{n} \\
\psi_{2}^{* *}=\frac{\pi}{\mathrm{n}+1} \mathrm{k}_{2}^{* *} \quad \mathrm{k}_{2}^{* *}=1, \ldots, \mathrm{n}
\end{gathered}
$$

It is convenient to assume that the quantity $\mathrm{n}$ is an even number (this condition does not limit the theory). In eq 11 sign + should be chosen at $\mathrm{I}=0$ and signs \pm at I $\neq 0$. The total number of values of the wave vector $\psi_{1}$ at a given phase shift between cells $\vec{\theta}=\left(\theta_{1}, \theta_{2}, \theta_{3}\right)$ is equal to $(n+1)$. Equations 11 and 12 indicate the existence of two branches in the relaxation spectrum of a network. The first branch corresponding to wave vector $\psi_{1}$ includes both intra- and interchain relaxation processes that are interconnected in a rather complicated way (eq 11). If I = 0 (see eq 11), the wave vector $\psi_{1}(\mathrm{l}=0) \equiv \psi_{\text {If }}$

$$
\begin{array}{r}
\psi_{\text {If }}\left(\theta_{1}, \theta_{2}, \theta_{3}\right)=\frac{1}{\mathrm{n}+1} \arccos \frac{1}{3}\left(\cos \theta_{1}+\cos \theta_{2}+\right. \\
\left.\cos \theta_{3}\right)
\end{array}
$$

describes only interchain low-frequency motions. If I $\neq 0$, the intrachain motions appear. They depend on the phase shift between network cells. Each intrachain mode $2 \mid \pi /(n+1)$ (see eq 11 ) is split into bands consisting of a great number of sublines. These sublines correspond to different phase shifts $\vec{\theta}$ between network cells. It should be noted that the broadening $\Delta \psi_{1}(\mathrm{I})=$ $\psi_{\text {If }}$ of intrachain modes is smaller than the difference $\left(\psi_{1}(I+1)-\psi_{1}(I)\right)$ between the neighboring $\psi_{1}(I)$ values. Therefore, the bands of neighboring intrachain modes are not superposed. The sets of wave vectors $\psi_{2}^{*}$ and $\psi_{2}^{* *}$ are intrachain quantities only and do not depend on the interchain wave vector $\vec{\theta}=\left(\theta_{1}, \theta_{2}, \theta_{3}\right)$. These wave vectors represent the purely intrachain branch of spectrum, which is doubly degenerated (see eq 12).

To construct normal modes (eq 4), it is necessary to combine the equations of motion (eq 2) and the boundary conditions in network junctions (eq 7). The determination of the form of the transformation from Cartesian coordinates $\mathrm{X}$ to normal modes $\mathrm{Q}$ is a rather complex problem. For this transformation we obtain 


$$
\begin{aligned}
& \mathrm{X}_{\mathrm{p}}(\Omega ; \mathrm{j} ; \mathrm{t}) \cong \sum_{\vec{\theta}, \psi_{1}} \exp \mathrm{i}(\vec{\Omega} \vec{\theta}) \times \\
& \exp \mathrm{i} \theta_{\mathrm{p}} \sin \mathrm{j} \psi_{1}+\sin (\mathrm{n}+1-\mathrm{j}) \psi_{1} \\
& \frac{\sin (\mathrm{n}+1) \psi_{1}}{\mathrm{Q}_{1}(\mathrm{t})+} \\
& \sum_{\vec{\theta}, \psi_{2}^{*}} \exp \mathrm{i}(\vec{\Omega} \vec{\theta}) \mathrm{f}_{\mathrm{p}}^{*}\left(\vec{\theta} ; \psi_{2}^{*}\right) \sin \mathrm{j} \psi_{2}^{*} \mathrm{Q}_{2}^{*}(\mathrm{t})+ \\
& \sum_{\vec{\theta}, \psi_{2}^{*}} \exp \mathrm{i}(\vec{\Omega} \vec{\theta}) \mathrm{f}_{\mathrm{p}}^{* *}\left(\vec{\theta} ; \psi_{2}^{* *}\right) \sin \mathrm{j} \psi_{2}^{* *} \mathrm{Q}_{2}^{* *}(\mathrm{t})
\end{aligned}
$$

Here $\mathrm{f}_{\mathrm{p}}^{*}\left(\vec{\theta} ; \psi_{2}^{*}\right)$ and $\mathrm{f}_{\mathrm{p}}^{* *}\left(\vec{\theta} ; \psi_{2}^{* *}\right)$ are functions of wave vectors $\psi$ and $\theta(p=1,2,3)$. These functions do not depend on index $\vec{\Omega}=(\alpha, \beta, \gamma)$ determining the position of the network cell and on the position of the bead $\mathrm{j}$ along the network chain. The functions $\mathrm{f}_{\mathrm{p}}^{*}\left(\vec{\theta} ; \psi_{2}^{*}\right)$ and $\mathrm{f}_{\mathrm{p}}^{* *}\left(\vec{\theta} ; \psi_{2}^{* *}\right)$ have a rather complicated form because of the multiple degeneration of this branch (the degeneration of every relaxation time of the branch is equal to $2 \mathrm{~N}^{3}$ for a cubic network system). Therefore, only the final results will be presented.

The normal coordinates of a system are separated into three sets $\left(\mathrm{Q}_{1}, \mathrm{Q}_{2}^{*}\right.$, and $\left.\mathrm{Q}_{2}^{* *}\right)$ corresponding to different branches of the spectrum. Normal modes $\mathrm{Q}_{1}\left(\vec{\theta} ; \psi_{1} ; \mathrm{t}\right)$ form the spectrum branch containing both theintra- and interchain motions. Normal modes $\mathrm{Q}_{2}^{*}\left(\vec{\theta} ; \psi_{2}^{*} ; \mathrm{t}\right)$ and $\mathrm{Q}_{2}^{* *}\left(\vec{\theta} ; \psi_{2}^{* *} ; \mathrm{t}\right)$ represent the doubly degenerated branch containing only intrachain motions (see above). One can see from eq 14 that this branch corresponds to the relaxation behavior of network chains with fixed junctions. This fact indicates that the approach developed by Mooney ${ }^{20}$ (who considered the linear Gaussian chain with fixed ends for describing the viscoel astic properties of cross-linked polymers in high-frequency region) can be related to the existence of a purely intrachain branch in the rigorous consideration of dynamics of a Gaussian network. It should be noted that the transformation from the Cartesian coordinates to normal modes for the network junctions has a simpler form because of the absence of the contribution of the intrachain degenerated branch to junction dynamics (see eq 14 at $j=0$ )

$$
\mathrm{X}_{0}(\Omega ; \mathrm{t}) \cong \frac{1}{\mathrm{~N}^{3 / 2}} \sqrt{\frac{1}{3(\mathrm{n}+1)}} \sum \exp \mathrm{i}\left(\vec{\Omega}, \psi_{1} \vec{\theta}\right) \mathrm{Q}_{1}\left(\vec{\theta} ; \psi_{1} ; \mathrm{t}\right)
$$

The normal modes (eqs 14 and 15) transform the dissipation function $\mathrm{R}(\mathrm{X})$ of a cubic Gaussian network

$$
R(X)=\frac{1}{2} \sum_{\Omega}\left[\zeta_{0} \sum_{p=1 j=1}^{3} \sum_{p}^{n} \dot{X}_{p}{ }^{2}(\Omega ; j ; t)+3 \xi_{0} \dot{X}_{0}{ }^{2}(\Omega ; t)\right]
$$

to the diagonal form with equal weights of all normal modes, i.e.,

$$
\mathrm{R}(\mathrm{Q})=\frac{1}{2} \zeta_{0} \sum_{\vec{\theta}} \sum_{\psi} \dot{\mathrm{Q}}^{2}(\vec{\theta} ; \psi ; \mathrm{t})
$$

Moreover, in this transformation the potential energy of a system

$$
U(X)=\frac{1}{2} K_{0} \sum_{\Omega} \sum_{p=1}^{3} \sum_{j=1}^{n+1}\left(X_{p}(\Omega ; j ; t)-X_{p}(\Omega ; j-1 ; t)\right)^{2}
$$

also becomes a diagonal form

$$
\mathrm{U}(\mathrm{Q})=\frac{1}{2} \mathrm{~K}_{0} \sum_{\vec{\theta}} \sum_{\psi} 2(1-\cos \psi) \mathrm{Q}^{2}(\vec{\theta} ; \psi ; \mathrm{t})
$$

It can be seen that the transformation from Cartesian coordinates to normal modes (eqs 14 and 15) is orthogonal but is not normalized because it represents both the rotation of Cartesian axes (when potential energy is transformed) and the scale stretching (when dissipation function is transformed) simultaneously. This scale stretching is related to the difference between friction constants of junctions and nonjunction beads (see eqs 8 and 16a).

It should be noted that the set of relaxation times of the network system (eq 5) corresponds to the following form of equations of motion for normal modes $\mathrm{Q}(\mathrm{t})$

$$
\rho(\mathrm{Q}) \xi_{0} \frac{\mathrm{d}}{\mathrm{dt}} \mathrm{Q}(\mathrm{t})+\rho(\mathrm{Q}) \mathrm{K}_{0} 2(1-\cos \psi) \mathrm{Q}(\mathrm{t})=\mathrm{F}_{\mathrm{Br}}(\mathrm{Q})
$$

where $\mathrm{F}_{\mathrm{Br}}(\mathrm{Q})$ are the components of stochastic Brownian force in normal coordinates. The quantity $\rho(\mathrm{Q})$ is equal to 3 at normal mode transformation of the equation of motion for the network junction (eq 2) and is equal to 1 if the equation of motion for a nonjunction bead (eq 1) is transformed. In contrast, the normal modes obtained (eqs 14 and 15) allow us to transform all equations of motion of network elements into the same form

$$
\xi_{0} \frac{\mathrm{d}}{\mathrm{dt}} \mathrm{Q}(\mathrm{t})+\mathrm{K}_{0} 2(1-\cos \psi) \mathrm{Q}(\mathrm{t})=\mathrm{F}_{\mathrm{Br}}(\mathrm{Q})
$$

These transformations from Cartesian coordinates to normal modes all ow various dynamic characteristics of the network system to be calculated.

2.3. Dynamic Characteristics of a Gaussian Network. Now we will consider the mean-square displacements of network junctions (cross-links) and of beads of network chains and the autocorrelation functions of subchain vectors and of end-to-end chain vectors between neighboring junctions. These characteristics can be manifested by different dynamic phenomena, for example, by dielectric and mechanical relaxation of cross-linked polymers, NMR phenomena, etc. The mean-square displacements as a function of time will be considered. These displacements describe translational diffusion of network junctions and nonjunction beads of multisegmental network chains. The meansquare displacement $\Delta \mathrm{X}^{2}(\mathrm{t})$ of a projection of the position vector of a network element on the $\mathrm{X}$ axis is determined as

$$
\Delta X^{2}(t)=\left\langle(X(t)-X(0))^{2}\right\rangle=2\left(\left\langle X^{2}\right\rangle-\langle X(0) X(t)\rangle\right)
$$

Here $\langle>$ means the configurational average. To calculate the quantity $\Delta \mathrm{X}^{2}(\mathrm{t})$, the transformation from Cartesian coordinates to normal modes (eqs 14 and 15) should be substituted into the expression for mean-square displacement (eq 20). The normal coordinates obtained are

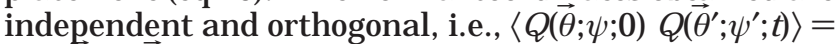
0 if $\theta \neq \vec{\theta}^{\prime}$ and (or) $\psi \neq \psi^{\prime}$. Therefore, the quantity $\Delta \mathrm{X}^{2}(\mathrm{t})$ includes only the autocorrelation functions of normal modes $\langle\mathrm{Q}(0) \mathrm{Q}(\mathrm{t})\rangle$, which have a simple time behavior

$$
\langle\mathrm{Q}(\vec{\theta} ; \psi ; 0) \mathrm{Q}(\vec{\theta} ; \psi ; \mathrm{t})\rangle=\left\langle\mathrm{Q}^{2}(\vec{\theta} ; \psi ; 0)\right\rangle \exp [-\mathrm{t} / \tau(\psi)]
$$


The law of equal distribution of energy according to classical degrees of freedom for a harmonic oscillator 21,22 must be applied to the equilibrium values of squares of normal modes $\left\langle\mathrm{Q}^{2}\right\rangle$, namely,

$$
\left\langle\mathrm{Q}^{2}(\vec{\theta} ; \psi ; 0)\right\rangle=\frac{\mathrm{k}_{\mathrm{B}} \mathrm{T}}{\mathrm{K}_{0} 2(1-\cos \psi)}
$$

where $\mathrm{K}_{0} 2(1-\cos \psi)$ are the eigenvalues of potential energy of a network (eq 17).

The consecutive use of this procedure for calculating the mean-square displacement of a nonjunction bead yields

$$
\begin{aligned}
& \Delta \mathrm{X}_{\mathrm{p}}^{2}(\Omega ; \mathrm{j} ; \mathrm{t})= \\
& \frac{\mathrm{k}_{\mathrm{B}} \mathrm{T}}{\mathrm{K}_{0}} \frac{2}{3 \mathrm{~N}^{3}(\mathrm{n}+1)} \sum_{\vec{\theta}} \sum_{\psi_{1}} \frac{1-\exp \left[-\mathrm{t} / \tau\left(\psi_{1}\right)\right]}{2\left(1-\cos \psi_{1}\right)}+ \\
& \mathrm{k}_{\mathrm{B}} \mathrm{T} \quad 1-\exp \left[-\mathrm{t} / \tau\left(\psi_{2}^{*}\right)\right]
\end{aligned}
$$

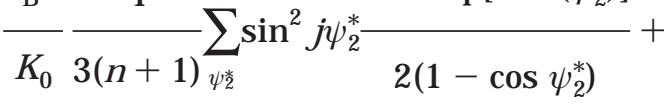

$$
\begin{aligned}
& \begin{array}{lll}
\mathrm{k}_{\mathrm{B}} \mathrm{T} & 4 & 1-\exp \left[-\mathrm{t} / \tau\left(\psi_{2}^{* *}\right)\right]
\end{array} \\
& \frac{\mathrm{K}}{\mathrm{K}_{0}} \frac{4(\mathrm{n}+1)}{\psi_{\psi_{2}^{*}}} \sin ^{2} \mathrm{j} \psi_{2}^{* *} \frac{}{2\left(1-\cos \psi_{2}^{* *}\right)}
\end{aligned}
$$

where index $\mathrm{p}$ means the number of a chain in the network cell $\Omega=(\alpha, \beta, \gamma)$. The sets of wave vectors $\psi_{1}$, $\psi_{2}^{*}$, and $\psi_{2}^{* *}$ are given by eqs 11 and 12 . It should be especially emphasized that the quantity $\psi_{1}$ is a function of phase shift $\vec{\theta}=\left(\theta_{1}, \theta_{2}, \theta_{3}\right)$ between the network cells (see eq 11). The contributions of wave vectors $\psi_{2}^{*}$ and $\psi_{2}^{* *}$ to the mean-square displacement of a network element (second and third terms in eq 23) have the same forms. Therefore, the expression for the mean-square displacement can be rewritten as

$$
\begin{aligned}
\Delta \mathrm{X}_{\mathrm{p}}{ }^{2}(\Omega ; \mathrm{j} ; \mathrm{t}) & = \\
& \frac{\mathrm{K}_{\mathrm{B}} T}{\mathrm{~K}_{0}} \frac{2}{3 \mathrm{~N}^{3}(\mathrm{n}+1)} \sum \sum_{\psi_{1}} \frac{1-\exp \left[-\mathrm{t} / \tau\left(\psi_{1}\right)\right]}{2\left(1-\cos \psi_{1}\right)}+ \\
& \frac{\mathrm{K}_{\mathrm{B}} T}{\mathrm{~K}_{0}} \frac{8}{3(\mathrm{n}+1)} \sum_{\psi_{2}} \sin ^{2} \mathrm{j} \psi_{2} \frac{1-\exp \left[-\mathrm{t} / \tau\left(\psi_{2}\right)\right]}{2\left(1-\cos \psi_{2}\right)}
\end{aligned}
$$

where the wave vector $\psi_{2}$ describes the purely intrachain branch of the relaxation spectrum and is determined as

$$
\psi_{2}=\psi_{2}^{*}=\psi_{2}^{* *}=\frac{\mathrm{k} \pi}{\mathrm{n}+1} \quad \mathrm{k}=1, \ldots, \mathrm{n}
$$

The mean-square displacements of network elements and other dynamic characteristics of a network (see below) do not depend on the position of the Gaussian chain in a cell (index p) because of the symmetry of a regular cubic system. The dynamic characteristics of a network do not depend on the three-dimensional index of a cell $\Omega=(\alpha, \beta, \gamma)$ because all the cells of a cubic network with periodic boundary conditions are equivalent. Therefore, the indices $p$ and $\Omega$ will be omitted below.

One can see from eq 24 that the mean-square displacements of non-junction beads of network chains depend on the bead position $\mathrm{j}$ along the chain $(\mathrm{j}=1, \ldots$, $n)$. This effect is related to the fact that a multisegmental Gaussian chain between neighboring network junctions is finite. In the case of finite Gaussian chains embedded in the network structure, the mean-square displacement of a non-junction bead, which is averaged over bead positions al ong the multisegmental chain, can be considered

$$
\Delta \mathrm{X}^{2}(\mathrm{t}) \equiv\left\langle\Delta \mathrm{X}^{2}(\mathrm{j} ; \mathrm{t})\right\rangle_{\mathrm{j}}=\frac{1}{\mathrm{n}_{\mathrm{j}=1}} \sum^{\mathrm{n}} \Delta \mathrm{X}^{2}(\mathrm{j} ; \mathrm{t})
$$

Using eq 24 we can write

$$
\begin{array}{r}
\Delta \mathrm{X}^{2}(\mathrm{t})=\frac{\mathrm{k}_{\mathrm{B}} T}{\mathrm{~K}_{0}} \frac{2}{3 \mathrm{~N}^{3}(\mathrm{n}+1) \vec{\theta} \sum_{\psi_{1}}} \frac{1-\exp \left[-\mathrm{t} / \tau\left(\psi_{1}\right)\right]}{2\left(1-\cos \psi_{1}\right)}+ \\
\frac{\mathrm{K}_{\mathrm{B}} T}{\mathrm{~K}_{0}} \frac{4}{3(\mathrm{n}+1)} \sum_{\psi_{2}} \frac{1-\exp \left[-\mathrm{t} / \tau\left(\psi_{2}\right)\right]}{2\left(1-\cos \psi_{2}\right)}
\end{array}
$$

The mean-square displacement of a network junction (see eq 24 at $\mathrm{j}=0$ ) is represented in a simpler form

$$
\Delta \mathrm{X}_{0}{ }^{2}(\mathrm{t})=\frac{\mathrm{k}_{\mathrm{B}} \mathrm{T}}{\mathrm{K}_{0}} \frac{2}{3 \mathrm{~N}^{3}(\mathrm{n}+1)} \sum_{\vec{\theta}} \sum_{\psi_{1}} \frac{1-\exp \left[-\mathrm{t} / \tau\left(\psi_{1}\right)\right]}{2\left(1-\cos \psi_{1}\right)}
$$

The time autocorrelation functions of the subchain vectors and of the end-to-end chain vectors between neighboring network junctions are also the important quantities characterizing the Brownian motion of the network. The projection of a subchain vector on the $\mathrm{X}$ axis is determined as

$$
\mathrm{u}_{\mathrm{x}}(\Omega ; \mathrm{j} ; \mathrm{t})=\mathrm{X}(\Omega ; \mathrm{j} ; \mathrm{t})-\mathrm{X}(\Omega ; \mathrm{j}-1 ; \mathrm{t})
$$

The projections of the end-to-end chain vectors between neighboring junctions have the following forms (there are three end-to-end chain vectors per cell of a cubic network)

$$
\begin{aligned}
& \mathrm{h}_{\mathrm{x} 1}(\alpha, \beta, \gamma ; \mathrm{t})=\mathrm{X}_{0}(\alpha, \beta, \gamma ; \mathrm{t})-\mathrm{x}_{0}(\alpha-1, \beta, \gamma ; \mathrm{t}) \\
& \mathrm{h}_{\mathrm{x} 2}(\alpha, \beta, \gamma ; \mathrm{t})=\mathrm{x}_{0}(\alpha, \beta, \gamma ; \mathrm{t})-\mathrm{x}_{0}(\alpha, \beta-1, \gamma ; \mathrm{t}) \\
& \mathrm{h}_{\mathrm{x} 3}(\alpha, \beta, \gamma ; \mathrm{t})=\mathrm{x}_{0}(\alpha, \beta, \gamma ; \mathrm{t})-\mathrm{x}_{0}(\alpha, \beta, \gamma-1 ; \mathrm{t})
\end{aligned}
$$

Using the above procedure for calculating the time autocorrelation functions $\mathrm{C}(\mathrm{u} ; \mathrm{t})=\langle\mathrm{u}(0) \mathrm{u}(\mathrm{t})\rangle$ and $\mathrm{C}(\mathrm{h} ; \mathrm{t})$ $=\langle\mathrm{h}(0) \mathrm{h}(\mathrm{t})\rangle$, we have

$$
\begin{aligned}
\mathrm{C}\left(\mathrm{u}_{\mathrm{x}}(\mathrm{j} ; \mathrm{t}) ; \mathrm{t}\right) & =\frac{\mathrm{k}_{\mathrm{B}} \mathrm{T}}{\mathrm{K}_{0}} \frac{1}{3 \mathrm{~N}^{3}(\mathrm{n}+1)} \sum_{\vec{\theta}} \sum_{\psi_{1}} \exp \left[-\mathrm{t} / \tau\left(\psi_{1}\right)\right]+ \\
\frac{\mathrm{k}_{\mathrm{B}} \mathrm{T}}{\mathrm{K}_{0}} & \frac{4}{3(\mathrm{n}+1)} \sum_{\psi_{2}} \cos ^{2}\left(\mathrm{j}-\frac{1}{2}\right) \psi_{2} \exp \left[-\mathrm{t} / \tau\left(\psi_{2}\right)\right]
\end{aligned}
$$




$$
\begin{gathered}
\mathrm{C}\left(\mathrm{h}_{\mathrm{x}} ; \mathrm{t}\right)=\frac{\mathrm{k}_{\mathrm{B}} \mathrm{T}}{\mathrm{K}_{0}} \frac{1}{9 \mathrm{~N}^{3}(\mathrm{n}+1) \vec{\theta}} \sum_{\psi_{1}} \exp \left[-\mathrm{t} / \tau\left(\psi_{1}\right)\right] \\
{\left[\frac{3-\cos \theta_{1}-\cos \theta_{2}-\cos \theta_{3}}{1-\cos \Psi_{1}}\right]}
\end{gathered}
$$

The autocorrelation function of a subchain vector $\mathrm{C}\left(\mathrm{u}_{\mathrm{x}}(\mathrm{j} ; \mathrm{t}) ; \mathrm{t}\right)$ depends on the position of a subchain along the multisegmental chain between network junctions $(j=1, \ldots, n+1)$. It is convenient to consider also the autocorrelation function $\mathrm{C}\left(\mathrm{u}_{\mathrm{x}} ; \mathrm{t}\right)$ averaged over subchain positions along the network chain

$$
\begin{aligned}
\mathrm{C}\left(\mathrm{u}_{\mathrm{x}} ; \mathrm{t}\right) \equiv & \frac{1}{\mathrm{n}+1} \sum_{\mathrm{j}=1}^{\mathrm{n}+1} \mathrm{C}\left(\mathrm{u}_{\mathrm{x}}(\mathrm{j} ; \mathrm{t}) ; \mathrm{t}\right)= \\
& \frac{\mathrm{k}_{\mathrm{B}} \mathrm{T}}{\mathrm{K}_{0}(\mathrm{n}+1)}\left[\frac{1}{3 \mathrm{~N}^{3}} \sum_{\vec{\theta}} \sum_{\psi_{1}} \exp \left[-\mathrm{t} / \tau\left(\psi_{1}\right)\right]+\right. \\
& \left.\frac{2}{3} \sum_{\psi_{2}} \exp \left[-\mathrm{t} / \tau\left(\psi_{2}\right)\right]\right]
\end{aligned}
$$

The expressions of dynamic quantities obtained for a cubic Gaussian network are derived by rigorous calculation and, therefore, are applicable to the cubic network with arbitrary values of network parameters $\mathrm{n}$ and $\mathrm{N}$.

In addition to the local dynamic characteristics, the normal mode transformation obtained allows the macroscopic viscoelastic behavior of a polymer network to be considered. In general, the macroscopic linear viscoelasticity of a polymer network (dynamic modulus and viscosity, relaxation modulus, and compliance) should be the subject of a separate detailed discussion. As an example, the relaxation modulus $\mathrm{G}(\mathrm{t})$ of polymer networks will be considered in this paper.

In order to study the viscoelastic properties of a Gaussian network, it is necessary to consider the behavior of a system in the field of external (shear or Iongitudinal) forces creating the time-dependent vel ocity gradient in the effective viscous medium. ${ }^{19,21,23}$ The viscoelastic functions can be obtained in terms of the method of calculating the additional work that is made by the system as a result of the friction of polymer chains with respect to the perturbed viscous medium. ${ }^{21,23}$ Using this approach and the normal mode transformation (eqs 14 and 15), we obtained the following expression for relaxation modulus $\mathrm{G}(\mathrm{t})$ of a cubic network consisting of multisegmental Gaussian chains

$$
\begin{array}{r}
\mathrm{G}(\mathrm{t})=\mathrm{G}_{\mathrm{e}}+v \mathrm{k}_{\mathrm{B}} \mathrm{T}\left[\frac{2}{\mathrm{~N}^{3} \vec{\theta}} \sum_{\psi_{1}} \exp \left[-\mathrm{t} / \tau^{\prime}\left(\psi_{1}\right)\right]+\right. \\
\left.4 \sum_{\psi_{2}} \exp \left[-\mathrm{t} / \tau^{\prime}\left(\psi_{2}\right)\right]\right]
\end{array}
$$

where $\mathrm{G}_{\mathrm{e}}$ is the equilibrium modulus of the bulk polymer network and $v$ is the number of cross-links per unit volume. Wave vectors $\psi_{1}(\vec{\theta})$ and $\psi_{2}$ are given by eqs 11 and 25, respectively. The relaxation times $\tau^{\prime}=$ $\tau / 2$ are 2 times smaller than those of local dynamic characteristics of the network (see eq 5) because the spectrum of mean squares of normal modes $\left\langle\mathrm{Q}^{2}\right\rangle$ is excited. $3,13,16$
It should be noted that the three-dimensional Gaussian network considered, which does not take into account the effects of excluded volume, has no static equilibrium dimension and must collapse to infinite density.4,24 In particular, the average values of position vectors of network elements and those of length vectors of subchains and network chains are equal to zero. In contrast, real cross-linked polymers are characterized by a certain fixed density (or dimension). According to J ames and Guth's approach ${ }^{25,26}$ an effective internal pressure may be introduced into the Gaussian network. This pressure stretches the network system and provides its finite volume. Recently, we applied this approach in the consideration of low-frequency dielectric and mechanical relaxation properties of polymer networks using a simplified coarse-grained network model. 15,16

If the network model is stretched by the effective internal pressure (or by the appropriate external force applied to network boundaries), a new characteristic parameter appears in the system, namely, the average length $h_{0}$ of the network chain between junctions (or the average length of the subchain: $\left.h_{0}=(n+1) u_{0}\right)$. This parameter is determined by effective internal pressure. Then the projection of the position vector of a network element $X(t)$ has to consist of the average value $\langle\mathrm{X}\rangle$ and the fluctuating part $\delta \mathrm{X}(\mathrm{t})$, i.e., $\mathrm{X}=\langle\mathrm{X}\rangle+$ $\delta \mathrm{X}$. The average values of projections of position vectors on the $X$ axis depend on the positions of network elements in the network, i.e., $\left\langle\mathrm{X}_{1}(\alpha, \beta, \gamma ; \mathrm{j})\right\rangle=\alpha \mathrm{h}_{0}+\mathrm{j} \mathrm{u}_{0}$ and $\left\langle\mathrm{X}_{2 ; 3}(\alpha, \beta, \gamma ; \mathrm{j})\right\rangle=\alpha \mathrm{h}_{0}$. One can see from eqs 1 and 2 that the equations of motion of network el ements do not change their forms but the projections of position vectors $X(t)$ should be replaced by the corresponding fluctuating part $\delta \mathrm{X}(\mathrm{t})$. The expressions for the mean-square displacements of network elements (eqs 24 and 28) are not changed either because $\left\langle(\mathrm{X}(\mathrm{t})-\mathrm{X}(0))^{2}\right\rangle$ is equal to $\langle(\delta \mathrm{X}(\mathrm{t})$ $\left.-\delta X(0))^{2}\right\rangle$ (see eq 20). In the consideration of relaxation of subchains and network chains it is necessary to consider the total autocorrelation functions $\langle u(0) u(t)\rangle$ $-\langle u\rangle^{2}$ and $\langle h(0) h(t)\rangle-\langle h\rangle^{2}$ instead of the quantities $\langle u(0) u(t)\rangle$ and $\langle h(0) h(t)\rangle$, respectively. F or these autocorrelation functions the expressions obtained (eqs 31 and 32) are valid (cf. ref 15).

It has been shown in refs 16 and 17 for the simplified coarse-grained model of a polymer network that the presence of the effective internal pressure, which stretches the Gaussian network and prevents its collapse, does not change the set of relaxation times, the shape of the relaxation spectrum, and the time dependence of the relaxation modulus of a network as compared with the "unstrained" network model. This conclusion may be easily extended to the network consisting of multisegmental Gaussian chains. Thus, the existence of the fixed average dimension of a polymer network does not affect the results obtained in this section.

\section{Discussion}

3.1. Relaxation Properties of Network J unctions. The dynamic characteristics of network junctions are determined by the wave vector $\psi_{1}$ only (purely intrachain branch $\psi_{2}$ is not included in these characteristics). It should be emphasized that the quantity $\psi_{1}$ depends on the phase shift $\vec{\theta}$ between the network cells in a complicated manner (eq 11). Therefore, the mean-square displacement of a network junction (eq 28) 
is determined by both the intra- and interchain relaxation processes because of the above dependence of $\psi_{1}$ on the phase shift between cells.

The problem of comparison of the contributions of intra- and interchain motions to dynamic properties is of great interest. The simplest way to estimate these contributions is to separate the relaxation processes with different scales of motions. The wave vector $\psi_{1}$ (eq 11) at I $=0$, which is equal to the quantity $\psi_{\text {If }}$ (eq 13), determines interchain large-scale motions only. The normal modes at $I \neq 0$ correspond to the contribution of intrachain motions to dynamic characteristics of a network. If I > 1, the first term in eq 11 is much greater than the second term depending on the phase shift $\vec{\theta}$ in the case of sufficiently long Gaussian chains between junctions. The relaxation times $\tau\left(\psi_{1}\right)$ at $\mathrm{I}>1$ are slightly dependent on the phase shift between cells. Therefore, to a first approximation which has a clear physical meaning, it can be assumed that the wave vector $\psi_{1}(I, \vec{\theta})$ at $I>1$ does not depend on the phase shift between the motions of network cells. Only when I = 1 are the first and second terms in eq 11 of the same order of magnitude, and the dependence of the wave vector $\psi_{1}(I=1)$ on the phase shift $\vec{\theta}$ cannot be neglected. Finally, we have the following approximate expressions for the wave vector $\psi_{1}$ in the case of sufficiently long Gaussian chains between cross-links

$$
\begin{aligned}
& \psi_{1}(\mathrm{I}=0)=\psi_{\text {If }}=\frac{1}{\mathrm{n}+1} \arccos \frac{1}{3}\left(\cos \theta_{1}+\cos \theta_{2}+\right. \\
& \left.\cos \theta_{3}\right) \\
& \psi_{1}(\mathrm{I}>1)=2 \mathrm{I} \pi /(\mathrm{n}+1) \quad \mathrm{I}=2, \ldots, \mathrm{n} / 2 \\
& \psi_{1}^{+}(\mathrm{I}=1)=2 \pi /(\mathrm{n}+1)+\psi_{\mathrm{If}}(\vec{\theta}) \\
& \psi_{1}^{-}(\mathrm{I}=1)=2 \pi /(\mathrm{n}+1)-\psi_{\mathrm{If}}(\vec{\theta})
\end{aligned}
$$

It should be noted that the set of wave vectors $\psi_{1}(\mid>1)$ is doubly degenerated because two signs \pm correspond to each value of I in eq 11.

Then the mean-square displacement of a network junction (eq 28) can be represented in the approximate form

$$
\begin{array}{r}
\Delta \mathrm{X}_{0}^{2}(\mathrm{t}) \cong \frac{\mathrm{k}_{\mathrm{B}} \mathrm{T}}{\mathrm{K}_{0}} \frac{2}{3(\mathrm{n}+1)} \frac{1}{\mathrm{~N}^{3}[}\left[\sum_{\vec{\theta}} \frac{1-\exp \left[-\mathrm{t} / \tau\left(\psi_{\mathrm{lf}}\right)\right]}{2\left(1-\cos \psi_{\mathrm{If}}\right)}+\right. \\
\sum_{\vec{\theta}} \frac{1-\exp \left[-\mathrm{t} / \tau\left(\psi_{1}^{+}\right)\right]}{2\left(1-\cos \psi_{1}^{+}\right)}+\sum_{\vec{\theta}} \frac{1-\exp \left[-\mathrm{t} / \tau\left(\psi_{1}^{-}\right)\right]}{2\left(1-\cos \psi_{1}^{-}\right)}+ \\
\left.2 \mathrm{~N}^{3} \sum_{\psi_{1}(\mathrm{l}>1)} \frac{1-\exp \left[-\mathrm{t} / \tau\left(\psi_{1}(\mathrm{l}>1)\right)\right]}{2\left(1-\cos \psi_{1}(\mathrm{l}>1)\right)}\right]
\end{array}
$$

The first term $(I=0)$ in brackets in eq 36 corresponds to the contribution of interchain large-scale motions to the mean-square displacements of network junctions. The second and third terms $(\mid= \pm 1)$ describe the contribution of the intrachain relaxation processes to the intermediate region between intra- and interchain parts of the spectrum. The normal modes corresponding to this intermediate region describe the intrachain motions of a "mixed" type, which take into account the correlation between the chains in the network. The fourth term $(\mid>1)$ corresponds to the intrachain contribution to the mean-square displacements. The normal modes that appear in this term correspond to a Gaussian chain with free ends because they determine the relaxation of network junctions.

Three terms in eq 36 are triple sums taken over components $\theta_{1}, \theta_{2}$, and $\theta_{3}$ of interchain wave vector $\vec{\theta}$. In order to simplify these terms, the components of wave vector $\vec{\theta}$ can be regarded as continuous variables in the case of a network with a great number of junctions ( $\mathrm{N}$ $\gg 1)$, and the long wave approximation ${ }^{15-17}$ is used $\left(\theta_{1,2,3}\right.$ $\ll \pi$ ). In this case the relaxation times of interchain cooperative motions $\tau_{\text {If }}$ and the relaxation times in the intermediate region $\left(\tau\left(\psi_{1}^{+}\right)\right.$and $\left.\tau\left(\psi_{1}^{-}\right)\right)$can be represented as

$$
\begin{gathered}
\tau_{\mathrm{If}} \cong 12(\mathrm{n}+1)^{2} \tau_{0}\left(1 / \theta^{2}\right) \\
\tau^{ \pm}=\tau\left(\psi_{1}^{ \pm}\right)=12 \tau_{0}(\mathrm{n}+1)^{2}(\theta \pm \mathrm{C})^{-2}
\end{gathered}
$$

where the constant $C$ is approximately equal to $2 \pi \sqrt{3}$. Here $\theta^{2}=\left(\theta_{1}\right)^{2}+\left(\theta_{2}\right)^{2}+\left(\theta_{3}\right)^{2}$ is the square of the interchain vector $\vec{\theta}$ and $\tau_{0}=\xi_{0} / 4 \mathrm{~K}_{0}$ is the relaxation time of a subchain. The quantity $\tau_{0}$ is the minimum relaxation time of the total spectrum of the network system (see eq 5 at $\psi=\pi$ ). Thus, the relaxation times $\tau_{\mid f}(\vec{\theta})$, $\tau^{+}(\vec{\theta})$, and $\tau^{-}(\theta)$ in the long wave approximation depend only on the square of the interchain wave vector $\vec{\theta}$. Therefore, it is convenient to introduce a spherical coordinate system into the triple integrals and to integrate over two variables except the radial distance from the center of the sphere $\left(\rho=\sqrt{\theta^{2}}\right)$. The fourth term in eq 36 corresponds to the contribution of intrachain motions and is the sum taken over wave vector $\psi_{1}(\mid>1)$, which does not depend (in the above approximation) on the phase shift $\vec{\theta}$ between network cells. The relaxation times of this intrachain contribution are finite and range from the relaxation time of the subchain $\tau_{0}$ to $\tau_{\max }^{0}$ (see eqs 5 and $35 \mathrm{~b}$ at $\mathrm{I}=2$ )

$$
\tau_{\max }^{0} \equiv \tau_{\max }\left(\psi_{1}(\mathrm{l}>1)\right)=\tau_{0}(\mathrm{n}+1)^{2}\left(1 / 4 \pi^{2}\right)
$$

One can see from rigorous expressions (eqs 5 and 11) for relaxation times that the relaxation spectrum consists of broad bands corresponding to the change of phase shift $\vec{\theta}$ at a given value of the intrachain mode $21 \pi /(n+1)$ (Figure $2 A)$. All intrachain bands $(I \neq 0)$ range from the relaxation time of subchain $\tau_{0}$ to the maximum relaxation time $\tau_{\text {chain }}$ of a network chain (see eq 5 at $\psi_{\text {chain }}^{\min } \cong \pi /(n+1)$ )

$$
\tau_{\text {chain }} \cong \tau_{0}(\mathrm{n}+1)^{2}\left(4 / \pi^{2}\right)
$$

Intrachain bands become more narrow with increasing values of I (Figure 2A). The interchain band $(I=0)$ extends to infinity and has the minimum relaxation time $\tau_{\mathrm{lf}}^{\mathrm{min}}$. It should be noted that the minimum relaxation time $\tau_{\mathrm{lf}}^{\min }$ of an interchain branch is found to be equal to the maximum relaxation time $\tau_{\text {chain }}$ of $a$ network chain

$$
\tau_{\mathrm{lf}}^{\min }=\tau_{\text {chain }}
$$

The above approximation leads to a certain change in the structure of relaxation times as compared with the rigorous spectrum. The minimum relaxation time 


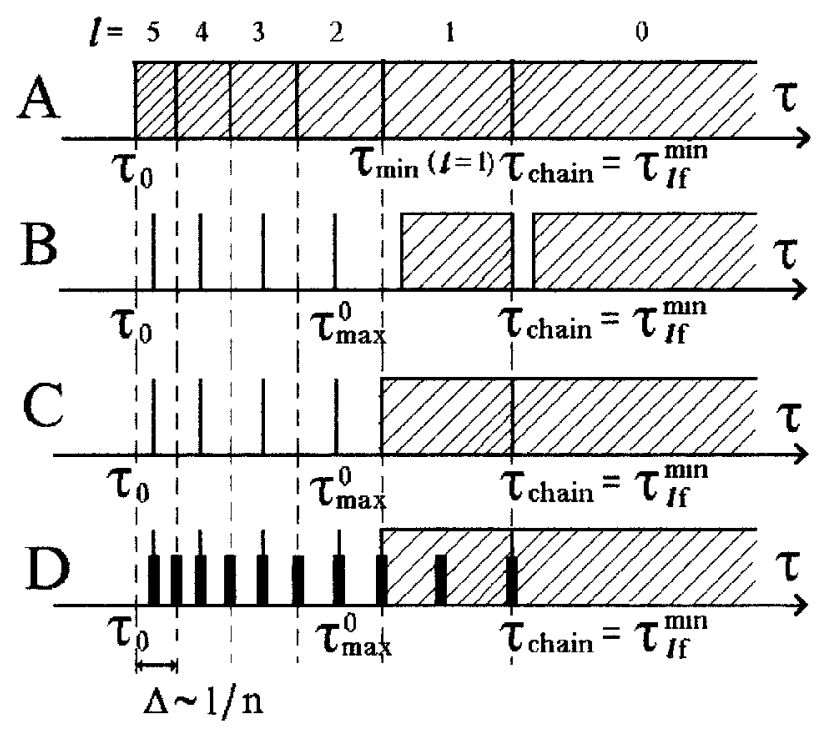

Figure 2. (A) Exact set of relaxation times for dynamic characteristics of junctions of a cubic Gaussian network ( $\mathrm{n}=$ 10). (B) Approximate set of relaxation times (see eqs 35 and 36 in the text). (C) Improved approximation for the set of relaxation times for dynamic characteristics of junctions. (D) I mproved approximation for the set of relaxation times for dynamic characteristics of nonjunction beads. The additional spectrum lines (vertical bars) appear, which correspond to the relaxation times of a Gaussian chain with fixed ends (see fifth term in eq 58).

of the interchain branch $(I=0)$ increases as compared with the rigorous quantity $\tau_{\mathrm{lf}}^{\min }$ (eq 41 ). In the intermediate region $(I=1)$ the maximum relaxation time $\tau_{\max }^{-}$remains equal to the maximum relaxation time $\tau_{\text {chain }}$ of a network chain, but the minimum relaxation time of this region $\tau_{\min }^{+}$increases. All other intrachain bands $(I>1)$ become lines (Figure $2 \mathrm{~B})$. The long wave approximation used for the spectrum bands at $I=0$ and I = 1 is a satisfactory approximation at $\theta_{1,2,3} \ll \pi$, but it gives the mi nimum relaxation times greater than rigorous values $\left(\theta_{1,2,3} \approx \pi\right)$. Therefore, the long wave approximation should be extrapolated to rigorous values of minimum relaxation times (Figure $2 \mathrm{C}$ ). This procedure reduces the changes of the relaxation spectrum because of the long wave approximation.

With the above approximation (eq 36), a comparison of the contributions of intra- and interchain relaxation processes to the mean-square displacement of a junction can be made for different time scales of motions. The initial slope of the time dependence of $\Delta \mathrm{X}_{0}{ }^{2}(\mathrm{t})$ can be easily obtained di rectly from rigorous eq 28 . Treating eq 28 at $\mathrm{t}<\tau_{0}$ we have

$$
\left.\Delta \mathrm{X}_{0}^{2}(\mathrm{t})\right|_{\mathrm{t}<\tau_{0}} \cong \frac{\mathrm{k}_{\mathrm{B}} \mathrm{T}}{\mathrm{K}_{0}} \frac{1}{6} \frac{\mathrm{t}}{\tau_{0}}
$$

Intrachain motions dominate in the initial slope of the mean-square displacement of the junction. The contribution of intrachain motions to the initial slope is greater than that of interchain cooperative motions by a factor of $(\mathrm{n}+1)$. In the time range from $\tau_{0}$ to $\tau_{\max }^{0}$ (eq 39) the mean-square displacement $\Delta \mathrm{X}_{0}^{2}(\mathrm{t})$ behaves as

$$
\Delta \mathrm{X}_{0}^{2}(\mathrm{t}) \cong \frac{2}{3 \pi} \frac{\mathrm{k}_{\mathrm{B}} \mathrm{T}}{\mathrm{K}_{0}} \sqrt{\frac{\mathrm{t}}{\tau_{0}}}
$$

This time dependence is typical of that for a bead of a

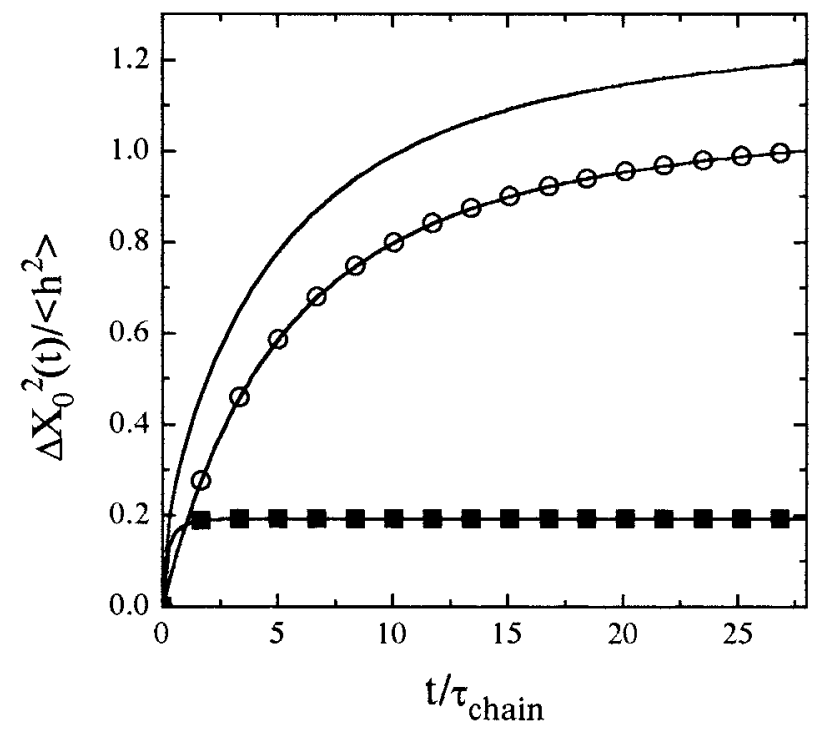

Figure 3. Reduced mean-square displacement of a network junction $\Delta \mathrm{X}_{0}^{2}(\mathrm{t}) /\left\langle\mathrm{h}^{2}\right\rangle$ versus rescaled time $\mathrm{t} / \tau_{\text {chain }}$ (solid line). Lines with open circles $(O)$ and solid squares $(\boldsymbol{\square})$ correspond to the contributions of inter- and intrachain relaxation processes, respectively. The quantity $\left\langle\mathrm{h}^{2}\right\rangle$ is the mean square of the end-to-end chain vector between neighboring junctions. Here $\mathrm{n}=20$.

long Gaussian chain. ${ }^{22,27,28}$ The intrachain motions dominate in this time range. At times greater than $\tau_{\max }^{0}$ (intermediate spectrum region) interchain cooperative motions begin to provide noticeable contribution, and the time behavior of $\Delta \mathrm{X}_{0}^{2}(\mathrm{t})$ changes

$$
\Delta \mathrm{X}_{0}^{2}(\mathrm{t}) \cong \frac{\mathrm{k}_{\mathrm{B}} \mathrm{T}}{\mathrm{K}_{0}}\left[\mathrm{C}_{1} \sqrt{\frac{\mathrm{t}}{\tau_{0}}}-\mathrm{C}_{2} \sqrt{\frac{\tau_{0}}{\mathrm{t}}}\right]
$$

where $\mathrm{C}_{1}$ and $\mathrm{C}_{2}$ are numerical constants. The contributions of intra- and interchain motions to the meansquare displacement of a network junction are approximately equal at $\mathrm{t} \cong \tau_{\mathrm{lf}}^{\min }=\tau_{\text {chain }}$ (Figure 3 ). At times greater than the minimum relaxation time of the interchain branch $\tau_{\mathrm{lf}}^{\mathrm{min}}$ the cooperative network motions provide the main contribution and $\Delta \mathrm{X}_{0}^{2}(\mathrm{t})$ has asymptotic behavior

$$
\begin{aligned}
\Delta \mathrm{X}_{0}{ }^{2}(\mathrm{t}) \cong \frac{\mathrm{K}_{\mathrm{B}} T}{\mathrm{~K}_{0}}\left[(\mathrm{n}+1)\left[\mathrm{C}_{\text {inter }}+\mathrm{C}_{\mathrm{intra}}\right]-\right. & \\
& \left.\frac{4(\mathrm{n}+1)^{2}}{\pi^{2} \sqrt{3}} \sqrt{\frac{\tau_{0}}{\mathrm{t}}}\right]
\end{aligned}
$$

where $\mathrm{C}_{\text {inter }}$ and $\mathrm{C}_{\text {intra }}$ are numerical constants corre sponding to the contributions of inter-and intrachain motions to the limiting value of $\Delta \mathrm{X}_{0}{ }^{2}(\mathrm{t})$. The limiting value of the mean-square displacement of a junction of cubic Gaussian network is finite, in agreement with previous network theories. ${ }^{4}$ The contribution of interchain relaxation processes to the limiting value $\left.\Delta \mathrm{X}_{0}^{2}\right|_{t \rightarrow \infty}$ is about 6 times greater than that of intrachain motions (see Figure 3). It should be noted that all curves in the figures are numerically calculated with the use of the rigorous expressions obtained for dynamic characteristics of a network. It is found that the ratio of contributions on different scales of motions is independent of the number of beads $\mathrm{n}$ in the multisegmental chain between network junctions $\left(C_{\text {inter }} / C_{\text {intra }} \approx 6\right)$. 
Similar estimations can also be made for the autocorrelation function of the end-to-end chain vector between neighboring network junctions (eq 32). Using numerical calculations, the equilibrium value of the mean-square distance $\left\langle h_{x}{ }^{2}\right\rangle$ averaged over all the network chains can be approximately represented in the form

$$
\begin{aligned}
\left\langle\mathrm{h}_{\mathrm{x}}{ }^{2}\right\rangle=\frac{1}{3 \mathrm{~N}^{3}} \sum_{\Omega}\left[\left\langle\mathrm{h}_{\mathrm{x} 1}{ }^{2}(\Omega ; 0)\right\rangle+\left\langle\mathrm{h}_{\mathrm{x} 2}{ }^{2}(\Omega ; 0)\right\rangle+\right. & \\
\left.\left\langle\mathrm{h}_{\mathrm{x} 3}{ }^{2}(\Omega ; 0)\right\rangle\right] & \cong \frac{1}{3} \frac{\mathrm{k}_{\mathrm{B}} \mathrm{T}}{\mathrm{K}_{0}}(\mathrm{n}+1)
\end{aligned}
$$

At short times (initial slope) the autocorrelation function of the end-to-end chain vector between neighboring junctions behaves as

$$
\mathrm{C}\left(\mathrm{h}_{\mathrm{x}} ; \mathrm{t}\right) \cong\left\langle\mathrm{h}_{\mathrm{x}}{ }^{2}\right\rangle-\frac{\mathrm{k}_{\mathrm{B}} \mathrm{T}}{\mathrm{K}_{0}} \frac{1}{6} \frac{\mathrm{t}}{\tau_{0}}
$$

The time dependence of the autocorrelation function $C\left(h_{x} ; t\right)=\left\langle h_{x}(t) h_{x}(0)\right\rangle$ at short times is determined by the intrachain motions. The contribution of the interchain cooperative spectrum to the total autocorrelation function $\mathrm{C}\left(\mathrm{h}_{\mathrm{x}} ; \mathrm{t}\right)$ (time-dependent part and equilibrium value of $\left.\left\langle h_{x}{ }^{2}\right\rangle\right)$ at short times is approximately 5 times greater than that of the intrachain spectrum. In the time range from $\tau_{0}$ to $\tau_{\max }^{0} \cong(1 / 16) \tau_{\text {chain }}$ (eq 39) the quantity $\mathrm{C}(\mathrm{h}$;t) behaves as

$$
C\left(h_{x} ; t\right) \cong\left\langle h_{x}^{2}\right\rangle-\frac{k_{B} T}{K_{0}} \frac{1}{3 \pi} \sqrt{\frac{t}{\tau_{0}}}
$$

The time dependence of $\mathrm{C}(\mathrm{h} ; \mathrm{t})$ is still determined by intrachain motions, whereas the total contribution of these motions to the quantity $\mathrm{C}(\mathrm{h}$;t) is much smaller than that of interchain relaxation processes. In the intermediate time range $\tau_{\max }^{0}<\mathrm{t}<\tau_{\mathrm{lf}}^{\min }$ the autocorrelation function behaves as

$$
C\left(h_{x} ; t\right) \cong\left\langle h_{x}^{2}\right\rangle-A * \frac{k_{B} T}{K_{0}} \sqrt{\frac{t}{\tau_{0}}}+A * * \frac{k_{B} T}{K_{0}}\left(\frac{\tau_{0}}{t}\right)^{3 / 2}
$$

where $A^{*}$ and $A^{* *}$ are the numerical constants. At times greater than the minimum relaxation time of interchain branch $\left(\tau_{\mathrm{lf}}^{\min }=\tau_{\text {chain }}\right), \mathrm{C}\left(\mathrm{h}_{\mathrm{x}}, \mathrm{t}\right)$ has behavior

$$
C\left(h_{x} ; t\right) \cong \frac{k_{B} T}{K_{0}} \frac{4(n+1)^{4}}{\pi^{2} \sqrt{3}}\left(\frac{\tau_{0}}{t}\right)^{3 / 2}
$$

i.e., it decreases to zero with increasing time. The interchain motions dominate, and their contribution is much greater than that of intrachain motions. The time dependence of the autocorrelation function $C\left(h_{x}, t\right)$ is plotted in Figure 4.

3.2. Comparison with a Simplified CoarseGrained Network Model. As mentioned in the Introduction, the interchain cooperative relaxation of network systems has usually been described by simplified network models in which intrachain motions are not taken into consideration. A comparison between the relaxation properties of a simplified coarse-grained model and a more realistic network model consisting of multisegmental Gaussian chains is of special importance. The coarse-grained model of a cubic network

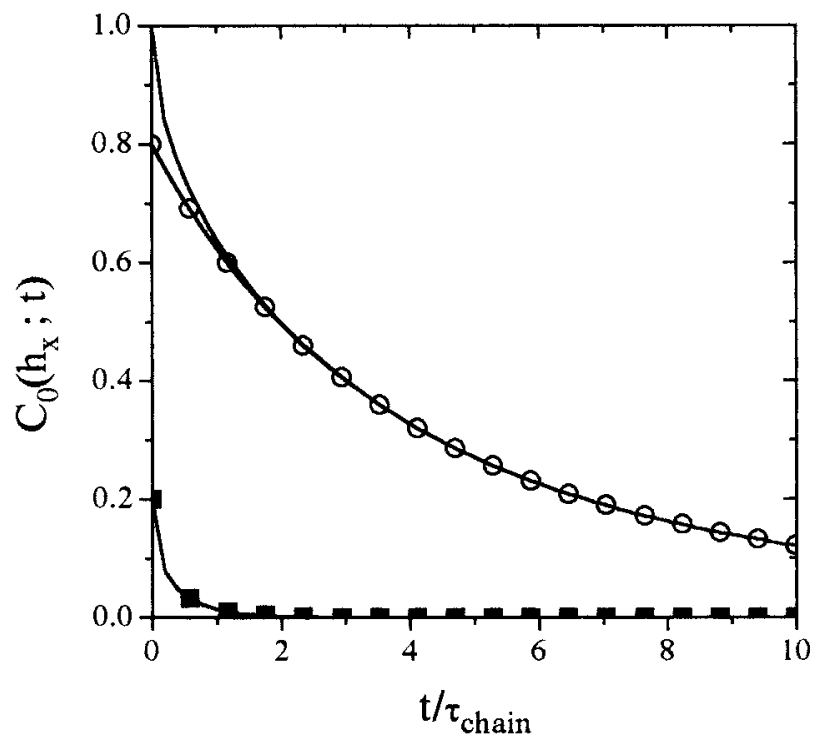

Figure 4. Normalized autocorrelation function $\mathrm{C}_{0}\left(\mathrm{~h}_{\mathrm{x}}, \mathrm{t}\right)$ of endto-end chain vector between neighboring junctions versus rescaled time $t / \tau_{\text {chain }}$ (solid line). Lines with open circles $(O)$ and solid squares ( $\boldsymbol{\square})$ correspond to the contributions of interand intrachain relaxation processes, respectively. The quantity $\mathrm{n}$ is equal to 20.

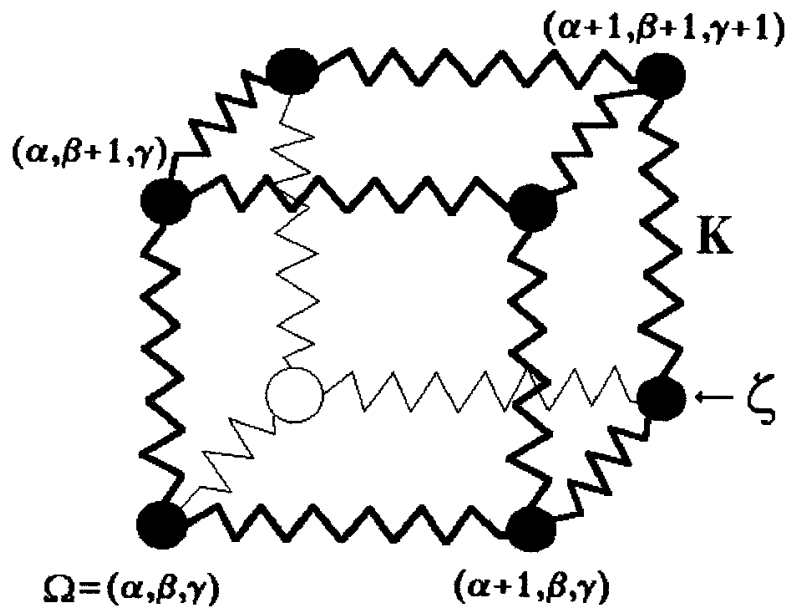

Figure 5. Elementary cell of a simplified coarse-grained cubic network.

consists of beads (junctions) and springs that connect beads into a unified regular network structure. ${ }^{13-17}$ The elasticity constant $\mathrm{K}$ of a spring between neighboring junctions corresponds to that of a Gaussian chain between these junctions. The friction constant $\xi$ of a junction (bead) describes the friction of chain fragments directly attached to a given junction (Figure 5). The relaxation times of a cubic coarse-grained network model consisting of $\mathrm{N}$ junctions along every Cartesian direction are determined by three-dimensional wave vector $\vec{\theta}$ corresponding to the phase shift between network cells ${ }^{14-17}$

$$
\tau(\vec{\theta})=\frac{\zeta}{2 \mathrm{~K}\left(3-\cos \theta_{1}-\cos \theta_{2}-\cos \theta_{3}\right)}
$$

If the periodic boundary conditions are used, the components of wave vector $\theta$ for a simplified network model have the same form as those for a network of multisegmental chains (eq 6). The minimum relaxation time of a coarse-grained network $\left(\theta_{1,2,3}=\pi\right)$ is equal to $\tau_{\min }=$ 
$\xi / 12 \mathrm{~K}$. F or large-scale motions $\left(\theta_{1,2,3} \ll \pi\right)$ the set of relaxation times for this model can be rewritten as

$$
\tau(\vec{\theta}) \cong 12 \tau_{\min }\left(1 / \theta^{2}\right)
$$

where $\theta^{2}=\left(\theta_{1}\right)^{2}+\left(\theta_{2}\right)^{2}+\left(\theta_{3}\right)^{2}$ is the square of wave vector $\vec{\theta}$.

If the motions on sufficiently large scales are considered, the sets of relaxation times of both network models correspond to the low-frequency cooperative rel axation only. Then the relaxation times of the cubic network of multisegmental Gaussian chains (eq 37) have to be equal to those of the simplified coarse-grained network (eq 52) in the large-scale region of motions. Therefore, the characteristic times of both network models should be related as follows

$$
\tau_{\min }=(\mathrm{n}+1)^{2} \tau_{0}
$$

Then the relation between the parameters (elasticity constant and friction constant) of both dynamic models of the network can easily be obtained. The elasticity constant $\mathrm{K}$ of a spring of a simplified coarse-grained network corresponds to the elasticity constant of the multisegmental chain as a whole, which contains ( $n+$ 1) subchains acting as springs with elasticity constants equal to $K_{0}$, i.e.,

$$
\mathrm{K}=\mathrm{K}_{0} /(\mathrm{n}+1)
$$

Then using eq 53 the relationship between the friction constants $\xi$ and $\xi_{0}$ can be obtained

$$
\zeta=3(n+1) \xi_{0}
$$

Hence, for an adequate description of the low-frequency relaxation behavior of a more realistic network model in terms of a simplified model, the friction of a junction of a coarse-grained network must be the sum of the frictions of the junction of the precise network model considered $\left(3 \xi_{0}\right)$ and of the halves of six chains directly attached to a given junction $\left(6(\mathrm{n} / 2) \xi_{0}\right)$.

For a coarse-grained network model, the autocorrelation function of the end-to-end chain vector between neighboring junctions and the mean-square displacement of a junction can be calculated using normal mode treatment ${ }^{15}$

$$
\begin{gathered}
\mathrm{C}\left(\mathrm{h}_{\mathrm{x}} ; \mathrm{t}\right)=\frac{1}{3} \frac{1}{\mathrm{~N}^{3}} \frac{\mathrm{k}_{\mathrm{B}} \mathrm{T}}{\mathrm{K}} \sum_{\vec{\theta}} \exp [-\mathrm{t} / \tau(\vec{\theta})] \\
\Delta \mathrm{X}^{2}(\mathrm{t})=\frac{2}{\mathrm{~N}^{3}} \frac{\mathrm{k}_{\mathrm{B}} \mathrm{T}}{\mathrm{K}} \sum_{\vec{\theta}} \frac{1-\exp [-\mathrm{t} / \tau(\vec{\theta})]}{2\left(3-\cos \theta_{1}-\cos \theta_{2}-\cos \theta_{3}\right)}
\end{gathered}
$$

With the long wave approximation it can be shown that the autocorrelation function $\mathrm{C}\left(\mathrm{h}_{\mathrm{x}}\right.$, $\left.\mathrm{t}\right)$ has asymptotic behavior $\left(4 / \sqrt{3} \pi^{2}\right)\left(\tau_{\mathrm{min}} / \mathrm{t}\right)^{3 / 2}\left(\mathrm{k}_{\mathrm{B}} \mathrm{T} / \mathrm{K}\right)$. Relationship 53 shows that the low-frequency tails of autocorrelation functions of both dynamic models of a network are completely equivalent. Similar arguments can be used for the large-scale behavior of the mean-square displacement of a junction. To sum up, the simplified coarse-grained network model provides a good fit to

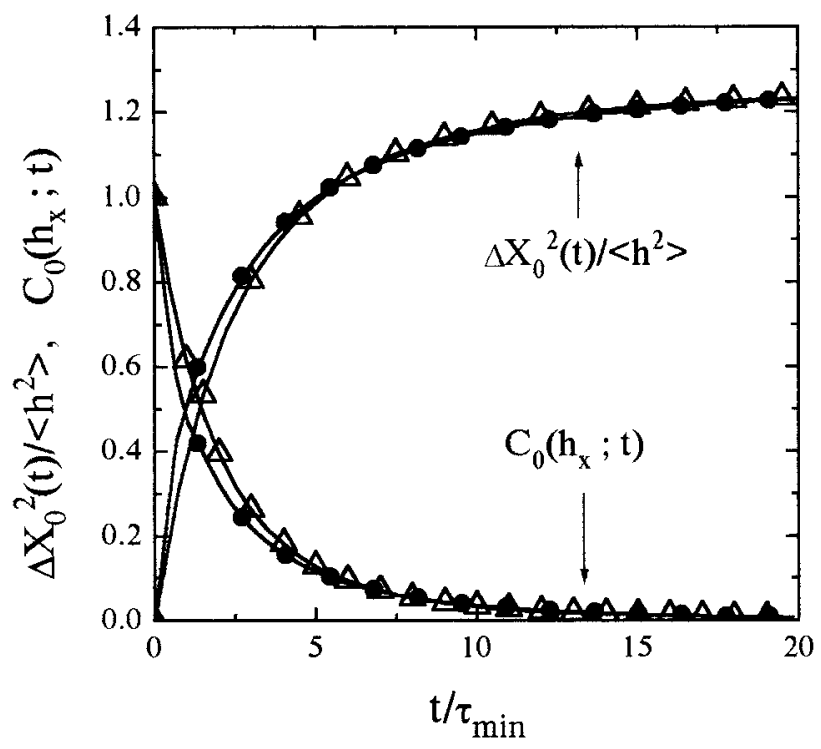

Figure 6. Reduced mean-square displacement of a network junction $\Delta \mathrm{X}_{0}^{2}(\mathrm{t}) /\left\langle\mathrm{h}^{2}\right\rangle$ and normalized autocorrelation function of end-to-end chain vector $\mathrm{C}_{0}\left(\mathrm{~h}_{\mathrm{x}} ; \mathrm{t}\right)$ versus rescaled time $\mathrm{t} / \tau_{\min }$ for a cubic network of multisegmental Gaussian chains (lines with solid circles $(\bullet)$ ) and for a simplified coarse-grained network (lines with open triangles $(\Delta)$ ).

interchain relaxation properties at times greater than the minimum relaxation time of this dynamic model (Figure 6).

It should be emphasized that in the limiting case ( $\mathrm{n}$ $=0$ ) of the network of multisegmental Gaussian chains, which corresponds to the coarse-grained network model, the set of relaxation times (eq 5) and the dynamic characteristics of junctions for the network of multisegmental chains (eqs 28 and 32) are exactly equivalent to those for the simplified network model (eqs 51, 56, and 57). Moreover, treating eq 56 at $t=0$ one can find that the equilibrium value of mean square $\left\langle h_{x}{ }^{2}\right\rangle_{\text {c.g. }}$. of the endto-end chain vector between neighboring junctions of a coarse-grained network is equal to $\mathrm{k}_{\mathrm{B}} \mathrm{T} / 3 \mathrm{~K}$. It can be seen that $\left\langle h_{x}{ }^{2}\right\rangle_{\text {c.g. }}$ is equal to that for a more precise network model (eq 46) if relationship 54 is taken into account. Thus, there is a complete agreement between the network model consisting of Gaussian multisegmental chains and the simplified coarse-grained network model at a proper relationship between the parameters of these dynamic models. Hence, the possibility of using the simplified network model for describing the low-frequency dynamic properties of networks is proved (at $\mathrm{t}>\tau_{\mathrm{min}}$ ).

3.3. Relaxation Properties of Beads of Network Chains. As compared with network junctions, the dynamic characteristics of nonjunction beads of a network are determined by wave vectors $\psi_{1}$ and $\psi_{2}$. In addition to the main branch described by wave vector $\psi_{1}$ (eq 11), the purely intrachain branch $\psi_{2}$ (eqs 12 and 25) corresponding to the relaxation of a network chain at fixed network junctions appears. Therefore, the contribution of intrachain motions to the relaxation characteristics increases as compared with those of junctions.

The approximate estimation applied in the consideration of relaxation behavior of junctions can be used for the analysis of relaxation properties of nonjunction beads. Equation 27 for the mean-square displacement of a nonjunction bead, which is averaged over the bead position along the chain, can be rewritten as 


$$
\begin{array}{r}
\Delta \mathrm{X}^{2}(\mathrm{t}) \cong \frac{\mathrm{k}_{\mathrm{B}} T}{\mathrm{~K}_{0}} \frac{2}{3(\mathrm{n}+1)} \frac{1}{\mathrm{~N}^{3}[}\left[\sum_{\vec{\theta}} \frac{1-\exp \left[-\mathrm{t} / \tau\left(\psi_{\mathrm{If}}\right)\right]}{2\left(1-\cos \psi_{\mathrm{If}}\right)}+\right. \\
\sum_{\vec{\theta}} \frac{1-\exp \left[-\mathrm{t} / \tau\left(\psi_{1}^{+}\right)\right]}{2\left(1-\cos \psi_{1}^{+}\right)}+\sum_{\vec{\theta}} \frac{1-\exp \left[-\mathrm{t} / \tau\left(\psi_{1}^{-}\right)\right]}{2\left(1-\cos \psi_{1}^{-}\right)}+ \\
2 \mathrm{~N}^{3} \sum_{\psi_{1}(\mathrm{I}>1)} \frac{1-\exp \left[-\mathrm{t} / \tau\left(\psi_{1}(\mathrm{l}>1)\right)\right]}{2\left(1-\cos \psi_{1}(\mathrm{l}>1)\right)}+ \\
\left.2 \mathrm{~N}^{3} \sum_{\psi_{2}}^{\frac{1-\exp \left[-\mathrm{t} / \tau\left(\psi_{2}\right)\right]}{2\left(1-\cos \psi_{2}\right)}}\right]
\end{array}
$$

A comparison of eqs 58 and 36 indicates that the contribution of intrachain motions in the case of nonjunction beads is greater than the analogous contribution for the network junctions. The additional term appears in eq 58. It is described by the intrachain wave vector $\psi_{2}$ (eq 25). This additional term has a similar form as the mean-square displacement of a bead of a linear Gaussian chain with fixed ends (see section 3.4.). The relaxation times of this intrachain term are finite and range from $\tau_{0}$ to the maximum relaxation time $\tau_{\text {chain }}$ (eq 40) equal to that of a Gaussian chain with fixed ends (Figure 2D).

The initial slope $\left(\mathrm{t} \ll \tau_{0}\right)$ of $\Delta \mathrm{X}^{2}(\mathrm{t})$ averaged over the bead position along the chain is given by

$$
\left.\Delta \mathrm{X}^{2}(\mathrm{t})\right|_{\mathrm{t}<\tau_{0}} \cong \frac{\mathrm{k}_{\mathrm{B}} \mathrm{T}}{\mathrm{K}_{0}} \frac{1}{2} \frac{\mathrm{t}}{\tau_{0}}
$$

It is 3 times greater than the analogous characteristic of the network junction (see eq 42). The contribution of intrachain motions dominates in the initial slope and is $3(n+1)$ times greater than that of interchain cooperative motions. In the time range from $\tau_{0}$ to $\tau_{\max }^{0}$ $\cong \tau_{\text {chain }} / 16$ (see eq 39 ) the intrachain relaxation processes provide the main contribution, and the quantity $\Delta \mathrm{X}^{2}(\mathrm{t})$ behaves as

$$
\Delta \mathrm{X}^{2}(\mathrm{t}) \cong \frac{\mathrm{k}_{\mathrm{B}} \mathrm{T}}{\mathrm{K}_{0}} \frac{2}{\pi} \sqrt{\frac{\mathrm{t}}{\tau_{0}}}
$$

The mean-square displacement of a bead of a network chain in this time range is also 3 times greater than that of the network junction (eq 43). In the time range from $\tau_{\max }^{0}$ to $\tau_{\text {chain }}$ (intermediate region) $\Delta \mathrm{X}^{2}(\mathrm{t})$ behaves as

$$
\Delta \mathrm{X}^{2}(\mathrm{t}) \cong \frac{\mathrm{k}_{\mathrm{B}} T}{\mathrm{~K}_{0}}\left[\mathrm{C}_{1}^{*} \sqrt{\frac{\mathrm{t}}{\tau_{0}}}-\mathrm{C}_{2}^{*} \sqrt{\frac{\tau_{0}}{\mathrm{t}}}\right]
$$

where $\mathrm{C}_{1}^{*}$ and $\mathrm{C}_{2}^{*}$ are numerical constants. Here the interchain collective motions begin to provide a noticeable contribution to the mean-square displacement of a bead. However, the intrachain motions still provide the main contribution in this time range. At times equal to the maximum relaxation time $\tau_{\text {chain }}$ of a chain between junctions, the contribution of intrachain motions is about 4 times greater than that of interchain cooperative motions. At times greater than $\tau_{\text {chain }}$ (which is equal to the minimum relaxation time $\tau_{\mathrm{lf}}^{\mathrm{min}}$ of interchain branch (eq 41)), the mean-square displacement

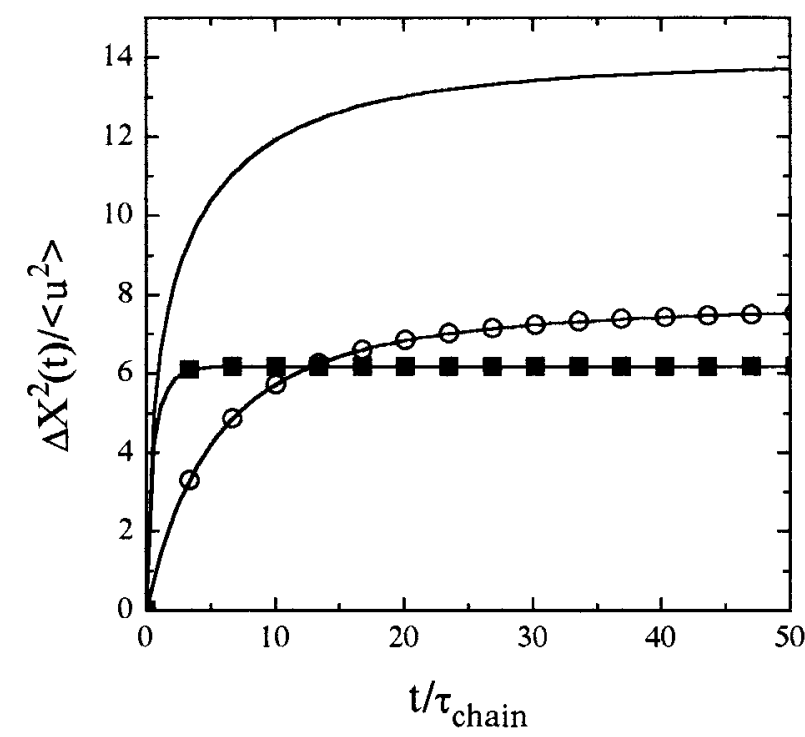

Figure 7. Reduced mean-square displacement of a nonjunction bead $\Delta \mathrm{X}^{2}(\mathrm{t}) /\left\langle\mathrm{u}^{2}\right\rangle$ versus rescaled time $t / \tau_{\text {chain }}$ (solid line). Lines with open circles $(O)$ and solid squares $(\boldsymbol{\square})$ correspond to the inter- and intrachain contributions, respectively. The quantity $\left\langle u^{2}\right\rangle$ is the mean square of subchain vector. Here $n=$ 20.

$\Delta \mathrm{X}^{2}(\mathrm{t})$ has asymptotic behavior

$\Delta X^{2}(t) \cong \frac{k_{B} T}{K_{0}}\left[(n+1)\left[C_{\text {inter }}^{*}+C_{\text {intra }}^{*}\right]-\frac{4(n+1)^{2}}{\pi^{2} \sqrt{3}} \sqrt{\frac{\tau_{0}}{t}}\right]$

where $\mathrm{C}_{\text {inter }}^{*}$ and $\mathrm{C}_{\text {intra }}^{*}$ are numerical constants corre sponding to the contributions of inter-and intrachain motions to the limiting value of $\Delta X^{2}(t)$. The time dependence of $\Delta \mathrm{X}^{2}(\mathrm{t})$ in this time range is determined by interchain motions. The contributions of the interand intrachain motions to $\Delta \mathrm{X}^{2}(\mathrm{t})$ become equal at $\mathrm{t} \cong$ $10 \tau_{\text {chain }}$ (Figure 7). This ratio is maintained up to the limiting value of the mean-square displacement $\left(C_{\text {inter }}\right.$ $\approx \mathrm{C}_{\text {intra }}$ ) and does not depend on the number of beads in the network chain.

The mean-square displacement of a nonjunction bead (as well as other local dynamic characteristics of network chains) depends on the bead position along the chain at sufficiently long times (t $>\tau_{\text {chain }}$ ). This dependence is completely determined by the purely intrachain branch $\psi_{2}$ (second term in eq 24). Treating eq 24 , one can estimate the increase in the limiting value of $\Delta X^{2}(t)$ as

$$
\left.\left.\Delta X^{2}(j)\right|_{t \rightarrow \infty} \cong \Delta X_{0}^{2}\right|_{t \rightarrow \infty}+\frac{k_{B} T}{K_{0}} \frac{4}{3} j\left(1-\frac{j}{n+1}\right)
$$

where $\left.\Delta \mathrm{X}_{0}^{2}\right|_{t \rightarrow \infty}$ is the limiting value of the mean-square displacement of a network junction. In Figure 8 the limiting values of the mean-square displacements are plotted versus the bead position $\mathrm{j}$ along the chain $(\mathrm{j}=$ $1, \ldots, n)$ for the case $n=20$. The bead in the middle of a chain "feels" the restrictions related to the inclusion into the network least of all. This middle bead has the highest diffusive mobility (Figure 9). In contrast, the network junction $(j=0)$ has the minimum limiting values of $\Delta X^{2}(t)$. Treating eq 63 , one can see that the limiting value of the mean-square displacement of a 


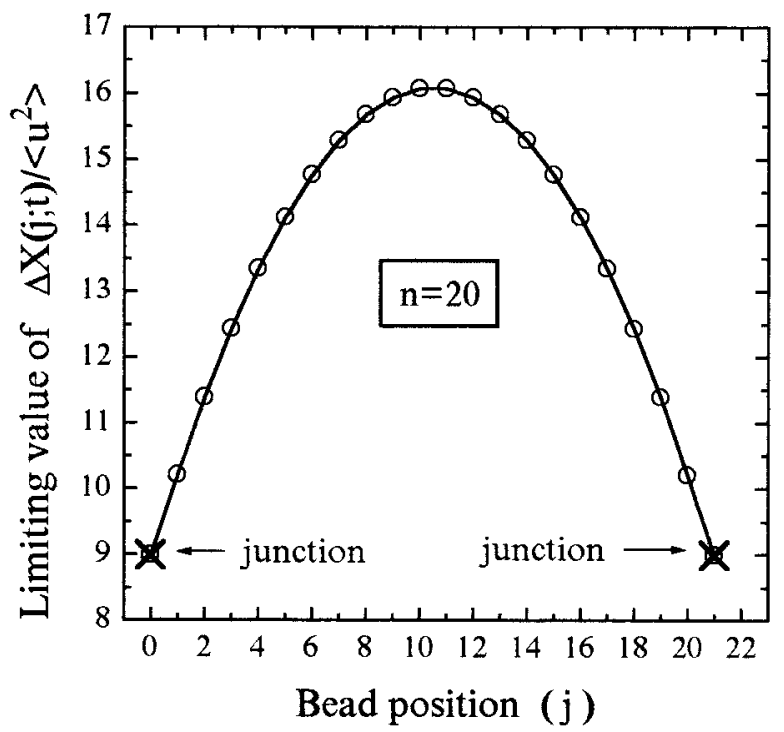

Figure 8. Limiting values of reduced mean-square displacements $\Delta \mathrm{X}^{2}(\mathrm{j}) /\left\langle\mathrm{u}^{2}\right\rangle$ for beads of a network chain versus bead position al ong the chain $(n=20)$. The case $\mathrm{j}=0$ corresponds to network junction (cross-link).

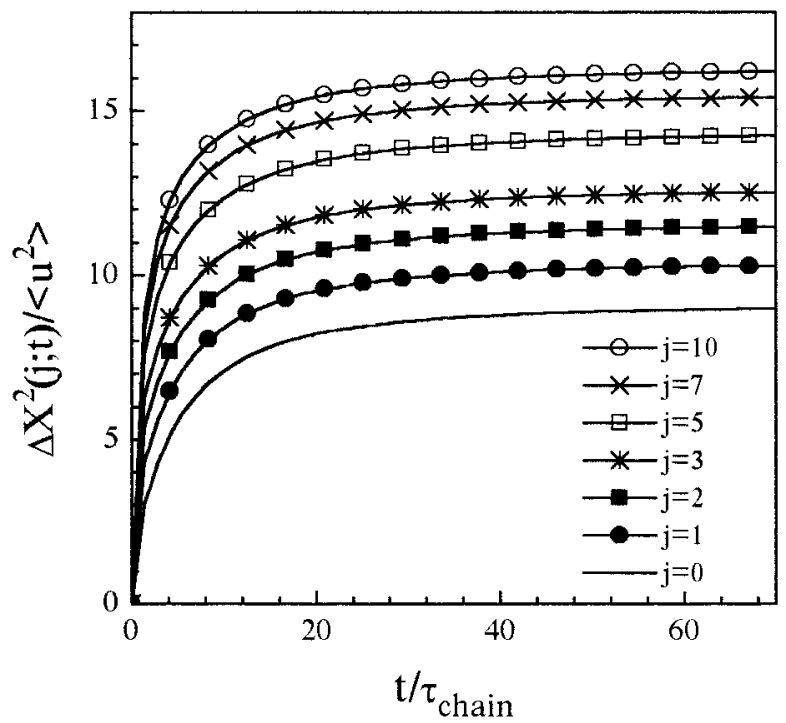

Figure 9. Reduced mean-square displacements $\Delta \mathrm{X}^{2}(\mathrm{j} ; \mathrm{t}) /\left\langle\mathrm{u}^{2}\right\rangle$ versus rescaled time $t / \tau_{\text {chain }}$ for different beads of a network chain $(n=20)$. Curve $\mathrm{j}=10$ corresponds to the middle bead of the network chain, and curve $\mathrm{j}=0$ corresponds to the junction.

junction (cross-link) is approximately 2 times smaller than the analogous characteristic of the middle bead (Figure 9).

The autocorrelation function $\mathrm{C}\left(\mathrm{u}_{\mathrm{x}} ; \mathrm{t}\right)$ averaged over the subchain position along the chain (eq 33) behaves at short times (initial slope) as

$$
\mathrm{C}\left(\mathrm{u}_{\mathrm{x}} ; \mathrm{t}\right) \cong\left\langle\mathrm{u}_{\mathrm{x}}{ }^{2}\right\rangle-\frac{\mathrm{k}_{\mathrm{B}} \mathrm{T}}{\mathrm{K}_{0}} \frac{1}{8} \frac{\mathrm{t}}{\tau_{0}}
$$

In the time range from $\tau_{0}$ to $\tau_{\max }^{0} \cong(1 / 16) \tau_{\text {chain, }}$ the quantity $\mathrm{C}\left(\mathrm{u}_{\mathrm{x}}, \mathrm{t}\right)$ decrease as

$$
C\left(u_{x} ; t\right) \cong \frac{k_{B} T}{K_{0}} \frac{2}{\pi} \sqrt{\frac{\tau_{0}}{t}}
$$

In the intermediate region $\left(\tau_{\max }^{0}<\mathrm{t}<\tau_{\text {chain }}\right)$ the time

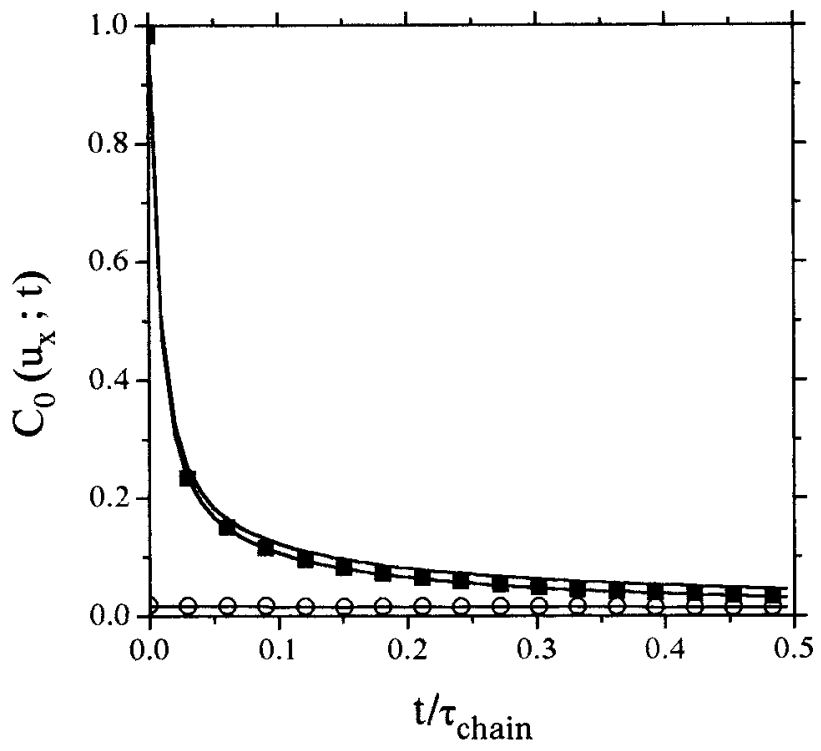

Figure 10. Normalized autocorrelation function $\mathrm{C}_{0}\left(\mathrm{u}_{\mathrm{x}} ; \mathrm{t}\right)$ of the subchain vector versus rescaled time $t / \tau_{\text {chain }}$ (solid line). Lines with open circles $(\bigcirc)$ and solid squares $(\square)$ correspond to the inter- and intrachain contributions, respectively. Here $n=20$.

behavior of $\mathrm{C}\left(\mathrm{u}_{\mathrm{x}}, \mathrm{t}\right)$ changes

$$
C\left(u_{x} ; t\right) \cong \frac{k_{B} T}{K_{0}}\left[A^{\prime} \sqrt{\frac{\tau_{0}}{t}}+A^{\prime \prime}\left(\frac{\tau_{0}}{t}\right)^{3 / 2}\right]
$$

where $A^{\prime}$ and $A^{\prime \prime}$ are numerical constants. The intrachain motions provide the main contribution to the autocorrelation function $\mathrm{C}\left(\mathrm{u}_{\mathrm{x}}, \mathrm{t}\right)$ up to the maximum relaxation time of a chain between network junctions $\tau_{\text {chain }}$ (Figure 10). In this time range the contribution of interchain relaxation processes is $(n+1)$ times smaller than that of intrachain motions. The interchain collective motions begin to be manifested at times greater than the maximum relaxation time of network

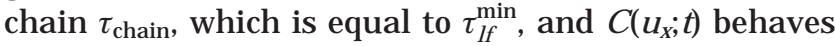
as

$$
C\left(u_{x} ; t\right) \cong \frac{k_{B} T}{K_{0}} \frac{4(n+1)^{2}}{\pi^{2} \sqrt{3}}\left(\frac{\tau_{0}}{t}\right)^{3 / 2}
$$

It should be emphasized that the autocorrelation function $\mathrm{C}\left(\mathrm{u}_{\mathrm{x}} ; \mathrm{t}\right)$ at $\mathrm{t}>\tau_{\text {chain }}$ decreases considerably (it becomes about $(n+1)$ times smaller than the equilibrium value $\left.\left\langle u_{x}{ }^{2}\right\rangle\right)$. Therefore, the influence of interchain relaxation processes on the quantity $\mathrm{C}\left(\mathrm{u}_{\mathrm{x}}, \mathrm{t}\right)$ is negligible in the time range from $\tau_{0}$ to $\tau_{\text {chain }}$ (Figure 10 ). The equilibrium value of the mean square of the subchain vector $\left\langle u_{x}{ }^{2}\right\rangle$ averaged over all the subchains of a network can be calculated using eq 31

$$
\begin{aligned}
\left\langle u_{x}{ }^{2}\right\rangle \equiv \frac{1}{N^{3}} \frac{1}{3(n+1)} \sum_{\Omega} \sum_{j=1}^{n+1} \sum_{p=1}^{3}\left\langle u_{p x}{ }^{2}(\Omega ; j)\right\rangle & = \\
& \frac{k_{B} T}{k_{0}}\left[1-\frac{2}{3(n+1)}\right]
\end{aligned}
$$

It should be noted that in the limiting case $\mathrm{n}=0$ the quantity $\left\langle u_{x}{ }^{2}\right\rangle$ corresponds to the equilibrium value of $\left\langle\mathrm{h}_{\mathrm{x}}{ }^{2}\right\rangle_{\text {c.g. }}$ for a simplified coarse-grained network and is equal to $\mathrm{K}_{\mathrm{B}} \mathrm{T} / 3 \mathrm{~K}_{0}$. I $\mathrm{n}$ contrast, in the limiting case $\mathrm{n} \rightarrow$ $\infty$, which corresponds to a linear Gaussian chain, $\left\langle u_{x}{ }^{2}\right\rangle$ 


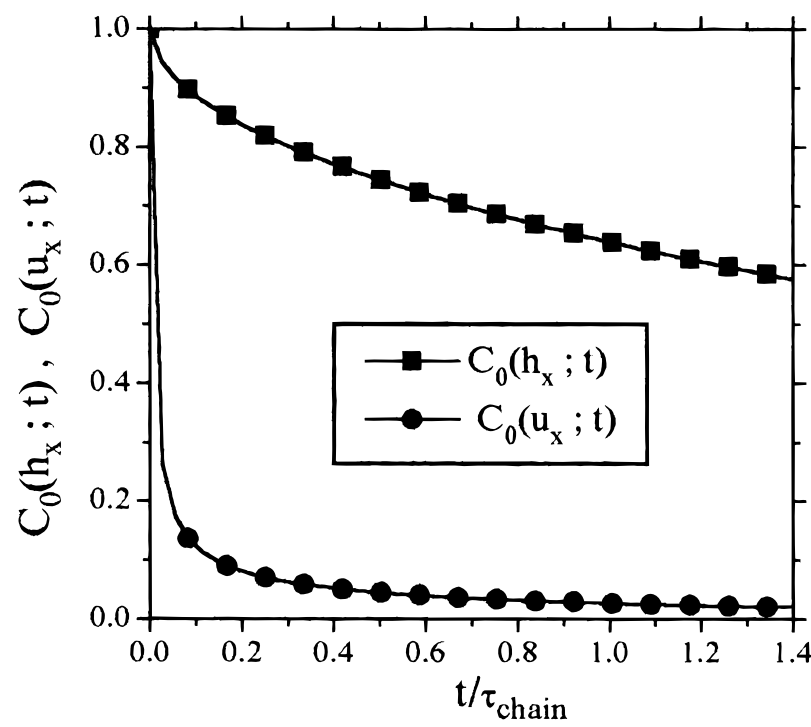

Figure 11. Normalized autocorrelation function $\mathrm{C}_{0}\left(\mathrm{~h}_{\mathrm{x}} ; \mathrm{t}\right)$ of the end-to-end chain vector between neighboring junctions $(\boldsymbol{\square})$ and the normalized autocorrelation function $\mathrm{C}_{0}\left(\mathrm{u}_{x}, \mathrm{t}\right)$ of the subchain vector $(\bullet)$ versus rescal ed time $t / \tau_{\text {chain }}(n=20)$.

is equal to $k_{B} T / K_{0}$, in agreement with the analogous equilibrium characteristic of a linear Gaussian chain. $21,22,28$

A comparison of the normalized autocorrelation function of the subchain vector $\left(\mathrm{C}_{0}\left(\mathrm{u}_{\mathrm{x}} ; \mathrm{t}\right) \cong \tilde{\tilde{\mathrm{A}}} \sqrt{\tau_{0} / \mathrm{t}}\right)$ and that of the end-to-end chain vector between the neighboring network junctions $\left(\mathrm{C}_{0}\left(\mathrm{~h}_{\mathrm{x}} ; \mathrm{t}\right) \cong 1-\tilde{\mathrm{A}}(\mathrm{n}+1)^{-1} \sqrt{\mathrm{t} / \tau_{0}}\right)$ in the time range from $\tau_{0}$ to $\tau_{\text {chain }}$ indicates that the decrease in $\mathrm{C}_{0}\left(\mathrm{~h}_{\mathrm{x}} ; \mathrm{t}\right)$ is much slower than that in the autocorrelation function $\mathrm{C}_{0}\left(\mathrm{u}_{\mathrm{x}} ; \mathrm{t}\right)$ (Figure 11). For example, at times having an order of magnitude equal to $\tau_{\text {chain, }}$ the autocorrelation function $\mathrm{C}_{0}\left(\mathrm{u}_{\mathrm{x}}, \mathrm{t}\right)$ is $(\mathrm{n}+1)$ times smaller than $\mathrm{C}_{0}\left(\mathrm{~h}_{\mathrm{x}} ; \mathrm{t}\right)$.

3.4. Comparison to a Gaussian Chain with Fixed Ends. As mentioned above, the dynamic properties of cross-linked polymers have been compared previously with those of linear chains with fixed ends ${ }^{19,20}$. Therefore, it is interesting to consider the relaxation properties of a chain with fixed ends. A comparison should be made between the intrachain dynamic characteristics (mean-square displacement of beads and autocorrelation functions of subchain vectors) of a network and a linear Gaussian chain. The dynamic characteristics of a Gaussian chain with fixed ends can be obtained directly using the normal mode treatment. The mean-square displacement of a bead of a Gaussian chain with fixed ends, which is averaged over the bead position along the chain, is given by

$$
\Delta \mathrm{X}^{2}(\mathrm{t})=\frac{2}{\mathrm{n}+1} \frac{\mathrm{k}_{\mathrm{B}} \mathrm{T}}{\mathrm{K}_{0}} \sum_{\psi} \frac{1-\exp [-\mathrm{t} / \tau(\psi)]}{2(1-\cos \psi)}
$$

Here the relaxation times $\tau(\psi)$ are determined by eq 5 , and the intrachain wave vector $\psi$ is equal to $k \pi /(n+1)$ where $\mathrm{k}=1, \ldots, \mathrm{n}$. It should be noted that $\psi$ cannot be equal to zero because the center of mass of the chain with fixed ends has no possibility to go into infinity. The relaxation times of a linear Gaussian chain with fixed ends are finite and range from $\tau_{0}$ to the maximum relaxation time of a chain $\tau_{\text {chain, }}$ which is given by eq 40.

A comparison of $\Delta X^{2}(t)$ of a bead of a linear chain (eq 69 ) with the analogous characteristic of a network chain

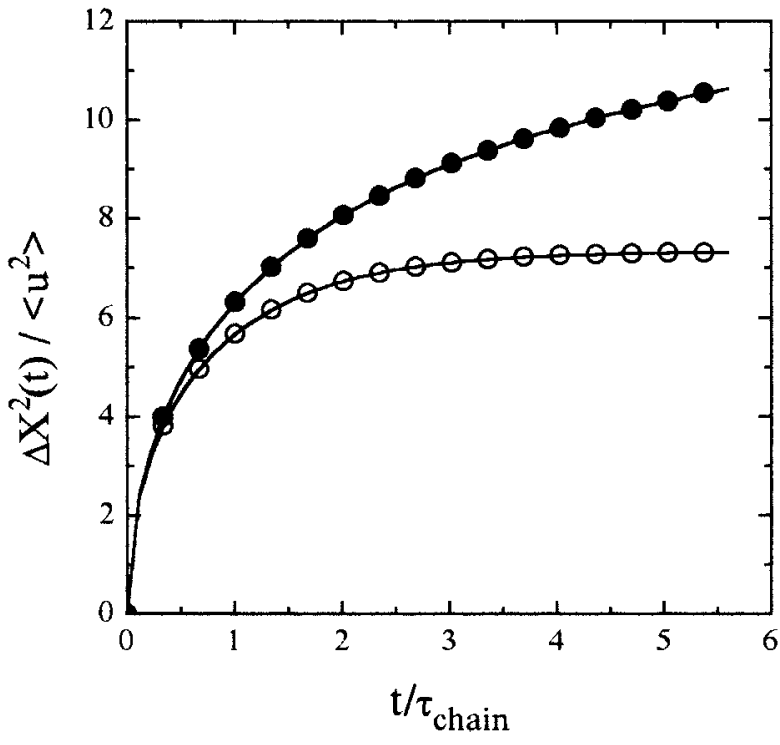

Figure 12. Reduced mean-square displacements $\Delta \mathrm{X}^{2}(\mathrm{t}) /\left\langle\mathrm{u}^{2}\right\rangle$ of beads of a network chain (line with solid circles $(\bullet)$ ) and of a Gaussian chain with fixed ends (line with open circles $(O)$ ) versus rescaled time $t / \tau_{\text {chain }}$ for the case $n=20$.

(eq 58) shows that the beads of linear chain and network chain have the same dynamic behavior when the intrachain motions in the network system dominate, i.e., at times smaller than $\tau_{\max }^{0} \cong \tau_{0}(\mathrm{n}+1)^{2} / 4 \pi^{2}$. For example, the initial slope of time dependence for the mean-square displacement of a bead of linear Gaussian chain is equal to $\left(k_{B} T / K_{0}\right)\left(t / 2 \tau_{0}\right)$, and the analogous characteristic of a bead of network chain has the same form (eq 59). At times greater than $\tau_{0}$ the quantity $\Delta \mathrm{X}^{2}(\mathrm{t})$ of a bead of a linear chain with fixed ends increases as $(2 / \pi)\left(\mathrm{k}_{\mathrm{B}} \mathrm{T} / \mathrm{K}_{0}\right)$. $\sqrt{\mathrm{t} / \tau_{0}}$ and tends to a finite limit at times with an order of magnitude equal to the maximum relaxation time of a chain $\tau_{\text {chain. }}$ In contrast to the linear Gaussian chain, the interchain large-scale motions begin to provide a noticeable contribution to the mean-square displace ment of a bead of a network chain at times greater than $\tau_{\max }^{0}$ (this time is smaller than $\tau_{\text {chain }}$ as mentioned above). Hence, the dynamic behavior of a bead of a network chain and that of a linear chain begin to differ at $\mathrm{t} \gg \tau_{\max }^{0} \cong(1 / 16) \tau_{\text {chain. }}$. The mean-square displacement of a bead of a chain with fixed ends reaches the limiting value at $\mathrm{t} \cong \tau_{\text {chain, }}$ whereas the mean-square displacement of a bead of a network chain still increases. It can be seen from Figure 12 that this difference becomes noticeable at $\mathrm{t} \cong 0.5 \tau_{\text {chain. }}$. The limiting value of $\Delta X^{2}(t)$ for a network chain is estimated to be 2 times greater than that for a chain with fixed ends.

Similar differences of relaxation behavior are manifested in the consideration of autocorrelation functions of subchain vectors $C\left(u_{x} ; t\right)$. The value of $C\left(u_{x} ; t\right)$ averaged over the subchain position along the chain with fixed ends has the form ${ }^{22}$

$$
\mathrm{C}\left(\mathrm{u}_{\mathrm{x}} ; \mathrm{t}\right)=\frac{1}{\mathrm{n}+1} \frac{\mathrm{k}_{\mathrm{B}} \mathrm{T}}{\mathrm{K}_{0}} \sum_{\psi} \exp [-\mathrm{t} / \tau(\psi)]
$$

At short times (initial slope) the autocorrelation function of the subchain vector of a chain with fixed ends has the same time behavior as that of a network chain, namely, $C\left(u_{x} ; t\right) \cong\left(k_{B} T / K_{0}\right)\left(1-t / 8 \tau_{0}\right)$. In the time range from $\tau_{0}$ to $\tau_{\text {chain }}$ the autocorrelation function $C\left(u_{x} ; t\right)$ for 
a linear Gaussian chain decreases as $\sqrt{\tau_{0} / t}$. At $t>$ $\tau_{\text {chain }}$ the quantity $\mathrm{C}\left(\mathrm{u}_{\mathrm{x}} ; \mathrm{t}\right)$ decreases as $\exp \left[-\mathrm{t} / \tau_{\text {chain }}\right]$ because the relaxation spectrum of a chain with fixed ends is finite. The autocorrelation function $\mathrm{C}\left(\mathrm{u}_{\mathrm{x}} ; \mathrm{t}\right)$ for a network chain has approximately the same behavior as that for a chain with fixed ends up to $t \cong \tau_{\text {chain. }}$. When $t>\tau_{\text {chain }}$ the autocorrelation function $\mathrm{C}\left(\mathrm{u}_{\mathrm{x}} ; \mathrm{t}\right)$ for $\mathrm{a}$ network chain decreases as $\left(\tau_{0} / t\right)^{3 / 2}$. Therefore, the time behavior of autocorrelation functions of subchain vectors for the chain with fixed ends and for the network chain begin to differ at $\mathrm{t}>\tau_{\text {chain. }}$. However, this difference in the time behavior appears when the normalized autocorrelation functions decrease considerably and become about $(n+1)$ times smaller than 1 .

3.5. Relaxation Modulus of a Gaussian Network. The estimation used in the consideration of local dynamic characteristics (section 3.1) can be applied to the analysis of the time dependence of relaxation modulus $\mathrm{G}(\mathrm{t})$ given by eq 34. At short times (from $\tau_{0}$ to $\tau_{\max }^{0} / 2$ (see eq 39)) the relaxation modulus behaves as

$$
\mathrm{G}(\mathrm{t}) \cong \mathrm{G}_{\mathrm{e}}+\frac{6 \sqrt{2}}{\pi} \nu \mathrm{k}_{\mathrm{B}} \mathrm{T}(\mathrm{n}+1)\left(\frac{\tau_{0}}{\mathrm{t}}\right)^{1 / 2}
$$

In this regi on the intrachain relaxation processes dominate in the relaxation modulus of a network. In the time range from $\tau_{\max }^{0} / 2$ to $\tau_{\text {chain }} / 2$ the cooperative interchain motions begin to be manifested. The time dependence of relaxation modulus $\mathrm{G}(\mathrm{t})$ changes

$$
\mathrm{G}(\mathrm{t}) \cong \mathrm{G}_{\mathrm{e}}+v \mathrm{k}_{\mathrm{B}} T\left[\tilde{\mathrm{C}}\left(\frac{\tau_{0}}{\mathrm{t}}\right)^{1 / 2}+\tilde{\tilde{C}}\left(\frac{\tau_{0}}{\mathrm{t}}\right)^{3 / 2}\right]
$$

Here $\tilde{C}$ and $\tilde{\tilde{C}}$ are numerical constants. However, the intrachain relaxation processes still provide the main contribution to the relaxation modulus $\mathrm{G}(\mathrm{t})$ of a network. The contributions of intra- and interchain motions become equal at times having an order of magnitude equal to $\tau_{\text {chain }} / 2$ (Figure 13). At times greater than $\tau_{\text {chain }} / 2$ the interchain relaxation processes dominate, and $\mathrm{G}(\mathrm{t})$ has asymptotic behavior

$$
\mathrm{G}(\mathrm{t}) \cong \mathrm{G}_{\mathrm{e}}+\frac{2 \sqrt{6}}{\pi^{2}} v \mathrm{k}_{\mathrm{B}} \mathrm{T}(\mathrm{n}+1)^{3}\left(\frac{\tau_{0}}{\mathrm{t}}\right)^{3 / 2}
$$

It should be noted that at times greater than $\tau_{\text {chain }} / 2$ the relaxati on modulus $\mathrm{G}(\mathrm{t})$ al ready becomes very small. At $\mathrm{t} \cong \tau_{\text {chain }} / 2$ the reduced quantity $\left(\mathrm{G}(\mathrm{t})-\mathrm{G}_{\mathrm{e}} /\left(\mathrm{G}(0)-\mathrm{G}_{\mathrm{e}}\right)\right.$ becomes about $3(n+1)$ times smaller than 1 (Figure 13).

The relaxation spectrum $\mathrm{H}(\tau)$ is determined as ${ }^{19}$

$$
\mathrm{G}(\mathrm{t})=\mathrm{G}_{\mathrm{e}}+\int \mathrm{H}(\tau) \exp [-\mathrm{t} / \tau] \mathrm{d} \ln \tau
$$

Its behavior may be easily estimated using the time dependence of the relaxation modulus. As mentioned above, the time dependence of $\mathrm{G}(\mathrm{t})$ is mostly governed by intrachain relaxation processes up to the longest relaxation time of a chain between junctions (see eqs 71 and 72). Hence, to a first approximation the relaxation spectrum $\mathrm{H}(\tau)$ behaves as $\mathrm{t}^{-1 / 2}$ at $\tau_{0} \ll \tau \ll \tau_{\text {chain }} / 2$. This behavior of the relaxation spectrum is similar to that of a long Gaussian chain. ${ }^{19}$ At times greater than $\tau_{\text {chain }} / 2$ the behavior of $\mathrm{G}(\mathrm{t})$ changes (see eq 73 ), and the relaxation spectrum $\mathrm{H}(\tau)$ has the corresponding asymptotic behavior $\tau^{-3 / 2}$ at $\tau \gg \tau_{\text {chain }} / 2$. This is typical of three-dimensional meshlike networks in the region of

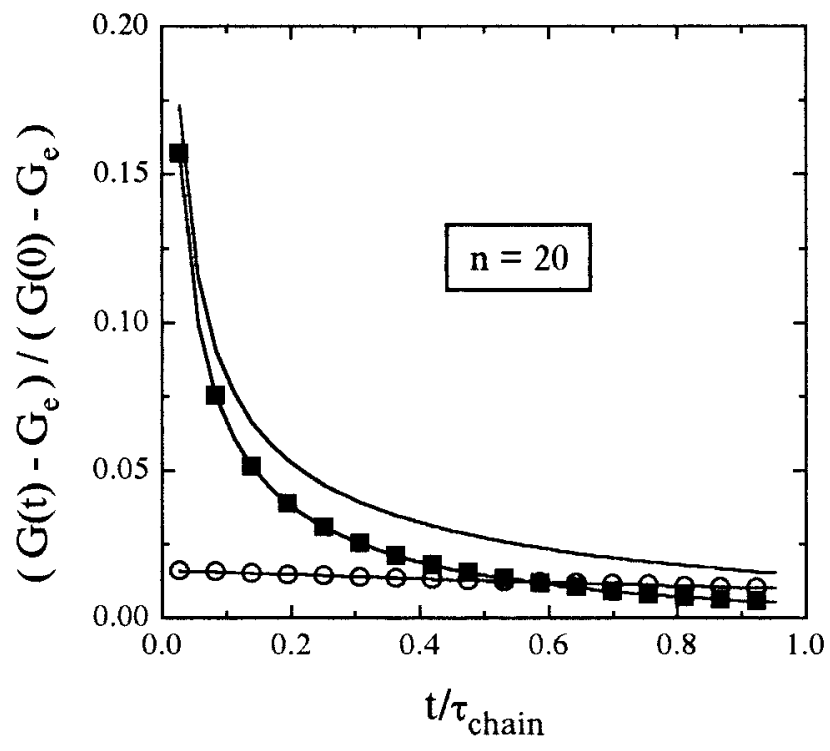

Figure 13. Reduced relaxation modulus $\left(\mathrm{G}(\mathrm{t})-\mathrm{G}_{\mathrm{e}}\right) /(\mathrm{G}(0)-$ $\mathrm{G}_{\mathrm{e}}$ ) versus rescaled time $t / \tau_{\text {chain }}$ (solid line) for the case $n=20$. Lines with open circles $(O)$ and solid squares $(\boldsymbol{\square})$ correspond to the inter- and intrachain contributions, respectively.

cooperative motions. ${ }^{1,3,14-17}$ It should be noted that the relaxation spectrum of a two-dimensional square network behaves as $\tau^{-1}$ in this region. ${ }^{5}$

To conclude, some remarks should be made about the differences in the spectra and relaxation moduli of the networks with meshlike and treelike topologies. The relaxation spectrum $\mathrm{H}(\tau)$ of a mesh like Gaussian network extends to infinity. The specific feature of the relaxation spectrum of a treelike network model ${ }^{11,12}$ is the existence of the finite maximum relaxation time $\tau_{\text {max. }}$ In the case of long multisegmental Gaussian chains the parts of the relaxation spectra of meshlike and treelike ${ }^{12}$ network models, which are determined by intrachain motions, are similar, and their behavior $\tau^{-1 / 2}$ is typical of a single Gaussian chain. It has been shown ${ }^{11}$ that the relaxation spectrum of a treelike network in the low-frequency region of cooperative chain motions behaves as $\tau^{-1 / 2}$, has root singularity in the vicinity of $\tau_{\max }$, and is cut off at $\tau>\tau_{\max }$. In contrast, the spectrum of the meshlike network model in the region of cooperative motions has a long wave tail, which extends to infinity and behaves as $\tau^{-3 / 2}$. This long wave tail of the relaxation spectrum appears in the time region where $\mathrm{G}(\mathrm{t})$ diminishes considerably (Figure 13). The relaxation moduli of meshlike and treelike network models have a qualitatively similar behavior $\tau^{-1 / 2}$ in the region of intrachain motions. The difference between the relaxation moduli of both network models are manifested in the region of cooperative motions where $\mathrm{G}(\mathrm{t})$ becomes very small. The noticeable difference in dynamic properties of meshlike and treelike networks may be expected in the consideration of dynamic modulus $\mathrm{G}^{\prime}(\omega)$ and viscosity $\eta^{\prime}(\omega)$ at low frequencies, i.e., in the region where the long wave tail of spectrum of meshlike network dominates. This is the subject of our further investigations.

\section{Conclusion}

To conclude, it can be said that the relaxation of regular polymer networks of long multisegmental Gaussian chains can be considered as a superposition of four different types of relaxation processes. The first type 
of these processes is related to the relaxation properties of network chains with fixed junctions. The second type corresponds to the relaxation behavior of linear chain with free ends. The third type determines the "intermediate" branch of the relaxation spectrum, which results from the splitting and broadening of the longest intrachain normal mode because of its interaction with the interchain relaxation modes. The fourth type of relaxation process is related to the long-scale cooperative interchain spectrum depending on the phase shift between network cells. The relative weights of these main types of relaxation processes depend on the dynamic characteristics considered. For example, the relaxation of network chains with fixed junctions (second type) does not provide the contribution to the relaxation properties of network junctions. In contrast, the dynamic relaxation properties of nonjunction beads are determined by all the above types of relaxation processes.

Acknowledgment. It is a pleasure to acknowledge the financial support of the Russian Foundation of Basic Research (Grants 96-03-33833 and 96-15-97401), Russian Federal Program Integration (Grant 326.38), I NTAS (Grant 93-2502-ext) and ISSE P (Grant a97-312 for A.A.G.).

\section{References and Notes}

(1) Ham, J . S. J . Chem. Phys. 1957, 26, 625.

(2) Takemura, T. J . Polym. Sci. 1958, 28, 185.

(3) Gotlib, Yu. Ya.; Salikhov K. M. Akusticheskii Zh. (Acoustic .) $1963,9,301$

(4) Ronca, G.; Allegra, G. J . Chem. Phys. 1975, 63, 4104.
(5) Chompff, A. J .; Duiser, J . A. J . Chem. Phys. 1966, 45, 1505

(6) Chompff, A. J.; Prins, W. J . J . Chem. Phys. 1968, 48, 235.

(7) Chompff, A. J . J . Chem. Phys. 1970, 53, 1566.

(8) Chompff, A. J J J Chem. Phys. 1970, 53, 1577.

(9) Ronca, G. Polymer 1979, 20, 1321.

(10) Ronca, G. J . Chem. Phys. 1980, 72 (1), 48.

(11) Graessley, W. W. Macromolecules 1980, 13, 372.

(12) Kloczkowski, A.; Mark, J. E.; Frisch H. L. Macromolecules $1990,23,3481$.

(13) Gotlib, Yu. Ya. Pure Appl. Chem. 1981, 53, 1531.

(14) Gotlib, Yu.; Golovachev, G. J . Non-Cryst. Solids 1994, 172, 850.

(15) Gotlib, Yu. Ya.; Gurtovenko, A. A. Macromol. Theory Simul. 1996, 5, 969.

(16) Gotlib, Yu. Ya.; Gurtovenko, A. A. Macromol. Theory Simul. 1997, 6, 523 .

(17) Gotlib, Yu.; Gurtovenko, A.; Golovachev G. In Chemical and Physical Networks; te Nijenhuis, K., Mijs, W., Eds.; The Wiley Polymer Networks Group Review Series, Volume One; J ohn Wiley \& Sons: Chichester, U.K., 1998; p 505.

(18) Rouse, P. E. J . Chem. Phys. 1953, 21, 1272.

(19) Ferry, J . D. Viscoel astic Properties of Polymers, 3rd ed.; J ohn Wiley and Sons: New York, 1980.

(20) Mooney, M. J . Polym. Sci. 1959, 34, 599

(21) Doi, M.; Edwards, S. F. The Theory of Polymer Dynamics; Clarendon Press: Oxford, U.K., 1986.

(22) Gotlib, Yu. Ya.; Darinsky, A. A.; Svetlov, Yu. E. Physical Kinetic of Macromolecules; Khimiya: Leningrad, 1986.

(23) Yamakawa, H. Modern Theory of Polymer Sol utions; Harper and Row: New York, 1971.

(24) J ames, H. M. J . Chem. Phys. 1947, 15, 651.

(25) J ames, H. M.; Guth, E. J . Chem. Phys. 1947, 15, 669.

(26) J ames, H. M.; Guth, E. J . Polym. Sci. 1949, 4, 153.

(27) de Gennes, P. G. Physics (Long Isl. Sity) 1967, 3, 37.

(28) Grosberg, A. Y.; Khokhlov, A. R. Statistical Physics of Macromolecules; AIP Press: New York, 1994.

MA980030A 